\title{
Ein geophysikalisches Eiszeit-Modell
}

\author{
Von H. Flohn, Bonn \\ Mit 8 Abbildungen
}

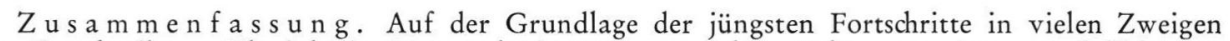
der Geophysik - Glaziologie, Meteorologie, Ozeanographie, Paläomagnetismus und Tektonophysik - wird ein synthetisches, rein geophysikalisches Modell der Klimaentwicklung im Tertiär und Pleistozän (mit Ausblicken auf das Permokarbon) entwickelt. Hierbei wird besonderes Gewicht auf die Abschätzung des Wärmehaushaltes der Ozeane gelegt; extraterrestrische Faktoren liefern höchstens einen sekundären Beitrag. Als Unterlage für eine weitere Diskussion wird eine knappe Zusammenstellung der wesentlichen Gesichtspunkte in Form von 10 Sätzen (Kapitel 6) gegeben.

A b s $\mathrm{r}$ a c t . Based on recent advances in many geophysical sciences - glaciology, meteorology, oceanography, paleomagnetics, tectonophysics - a synthetic geophysical Ice Age model is derived as a base for further discussion. Special attention is devoted to the heat budget of oceans.

The principal features of this model can be summarized as follows:

1) The primary prerequisite of the Pleistocene and Permo-Carboniferous glaciations is the drift of an isolated continental fragment - Antarctica in the Tertiary Era, Gondwanaland in Devon - into a circum-polar position. Two processes were involved in this drift in the Tertiary Era: a) convective currents in the earth's mantle (spreading of the ocean floor), and b) wandering of the earth's rotational axis relative to its mantle. The direction and speed of both these processes are ascertained from paleo-magnetic and (or) astronomical data to be a few $\mathrm{cm} / \mathrm{a}$.

2) Winter snow then builds up on the continent in the vicinity of the pole. Melting of this snow may lead to cooling by $1^{\circ} \mathrm{C}$ of an oceanic bottom water layer 1,000 meters thick in a period of $105-106 \mathrm{a}$. As a result of mixing processes this cooling affects all oceans - with a minimum effect in the surface layer in the tropical zone. This explains the simultaneous gradual cooling of the high latitudes and nearly constant temperature of the tropics during the Tertiary Era.

3) After the sea temperature has sunken to a bit over $0^{\circ} \mathrm{C}$, which happens at the latest in the Pliocene Era, a more or less stationary glaciation of the "warm" (mountain) type accumulates on Antarctica. Because of increasing cooling about the end of the Pliocene Era, this glaciation changes into the "cold" type. It has then a positive mass balance and causes an eustatic sinking of the sea level. Glaciers in the polar mountains of the northern continental areas also form.

4) As soon as the antarctic ice cap reaches the critical thickness at which increasing pressure causes melting on the lower surface, surges (in the sense of A. T. Wilsons theory) begin. These (gradual) surges cause: a) the formation of large ice shelves on the edges of Antarctica to about $55^{\circ} \mathrm{S}$ lat. and b) a rise in the sea level of 20 to $30 \mathrm{~m}$. An increase of surface albedo leads to a global cooling of about $5^{\circ} \mathrm{C}$, the formation of ice-sheets in areas of the northern continents, and a sinking of the sea level ( $\max . \mathrm{ca} .-145 \mathrm{~m}$ ).

5) After the onset of pressure release, the antarctic ice cap stabilizes at a lesser thickness. The shelf ice gradually disintegrates because of lack of supply.

6) The cooling of the water and air currents from both north and south leads to an advective lowering of the ocean temperature of about 5 to $6^{\circ} \mathrm{C}$ in the tropics, to a reduction in evaporation of about $30 \%$, and, under stationary conditions, to a global arid phase at the height of the glacial period.

7) In this arid phase, all ice caps slow their rate of growth. Loess dust covers the ice caps of the northern continents (excluding Greenland and Antarctica) and increases summer melting. The transition to disintegration, with the accompanying eustatic sea level rise, begins.

8) The quasi-periodical events under 4) -7) have, together, a periodicity of about 30.000 to 50.000 a. Long-periodic changes of the earth's orbit may, but do not necessarily, contribute to this cycle.

9) The much greater glaciation of the Atlantic sector of the northern continents is interpreted primarily as a result of the effect of the antarctic shelf ice surges which reach farther into the South Atlantic, and secondarily as a result of the formation of a land bridge in the Bering Sea which hinders mass and heat exchange between the Pacific and Arctic Oceans.

10) The apparent lack of similar quasi-periodic events in the Permo-Carboniferous Era might be interpreted as an effect of the great size of the Gondwana Continent which does not allow the building of shelf ice. 
This model is, as far as we can see, in no way contradictory to either the observed facts or the complicated physical inter-relations involved which appears as an advantage over many "extraterrestrial" Ice Age theories. It needs neither a change of the solar constant, nor, necessarily, an influence of a changing orbit of the earth; neither a change in gravitation nor, with the exception of the temperature-dependent constituent of water-vapor, a change in the composition of the earth's atmosphere.

\section{Allgemeine Voraussetzungen}

Der Komplex der pleistozänen Klimaschwankungen, mit einem mindestens achtmaligen Wechsel zwischen Kaltzeiten und Warmzeiten mit einer quasiperiodischen Zeitskala von 40-50000 a (= Jahre), hat in den letzten Jahren über die bisher beteiligten Disziplinen der Erdgeschichte (und ihrer Anwendungen in Archäologie und Vorgeschichte) hinaus wachsendes Interesse im Rahmen der exakten Naturwissenschaften gefunden. Geophysiker, Meteorologen und Astrophysiker, aber auch Physiker und Chemiker werden in wachsendem Ausmaß von den Möglichkeiten angezogen, die sich aus der Entwicklung ihrer eigenen Fächer zur Lösung dieses vielschichtigen Haupt-Problems der Paläoklimatologie anbieten. Das hier zur Diskussion gestellte Modell berücksichtigt - soweit dem Einzelnen noch überschaubar - diese neuesten Entwicklungen zu dem Versuch einer Synthese. Es geht aus von empirischen Befunden - wobei allerdings eine Reihe von ungelösten Problemen der Zeitfolge und Datierung notwendig offen gelassen werden müssen - und versucht, aus zahlreichen Ergebnissen der neueren Geophysik (Glaziologie, Meteorologie, Ozeanographie, Tektonophysik und Erdmagnetismus), in Umrissen ein widerspruchsfreies und konsistentes Gesamtbild zu skizzieren. Die geophysikalischen Voraussetzungen hat auch schon Fairbridge $(1961,1967)$ erörtert; als entscheidende Ergänzung steht hier die Diskussion des Wärmehaushaltes im Vordergrund.

Im Gegensatz zu den meisten bisherigen Versuchen sollen extraterrestrische Hypothesen nicht mitberücksichtigt werden. Dies gilt zunächst für die vieldiskutierte Rolle der Erdbahnelemente nach Milankowitch (1938), bzw. in revidierter Form nach van WoeRком (1953), sowie für die Tropenzone (BERNARd 1962). Trotz aller - vom Verfasser bisher geteilten (1959) - Einwände (ScHWARzBACH 1961) darf man diesen Effekt nicht a priori ausschließen. Doch zeigt eine quantitative Diskussion, daß nur in hohen Breiten die Variation der Schiefe der Ekliptik einen wirksamen Beitrag zum Strahlungshaushalt liefert (vgl. hierzu SHaw \& Donn 1968). Die zeitliche Übereinstimmung dieser Periode (von rund 40000 a) mit den beobachteten Klimaschwankungen des mittleren und jüngeren Pleistozäns (Emiliani 1955, 1957, 1961, 1966 u. Broecker 1966, 1968) ist ein ernsthaftes und nicht ohne weiteres widerlegbares Argument. Unser Modell benötigt diesen Effekt zwar nicht, schließt aber andererseits eine unterstützende Rolle nicht aus.

Die astrophysikalischen Hypothesen - genannt sei nur die Variabilität der Sonne entweder im (energiereichsten) sichtbaren Spektralbereich (OOPIK 1965) oder im kurzwelligen Ultraviolett (FLOHN 1952) - sollen hier bewußt vernachlässigt werden. Sie sind zunächst heute (noch) unbeweisbar; vieles spricht dafür, daß die Zeitskala (ÖPIK 1965 u. Mitchell jr. 1965) wirksamer Prozesse astrophysikalischer Natur mindestens bei $10^{6}-10^{7}$ a liegt, so daß die zeitliche Untergliederung des Pleistozäns (mit ihrer Zeitskala von $10^{4}-10^{5}$ a) nicht auf diese langsameren Vorgänge zurückgeführt werden kann. Ähnliches gilt erst recht für die höchst interessante Hypothese von DiRAC-JORDAN (1966) einer sehr langsamen Abnahme der Gravitationskonstanten, deren Zeitskala (109-1010 a) noch wesentlich größer ist; auf ihre mögliche geophysikalisch-paläoklimatische Rolle soll bei anderer Gelegenheit eingegangen werden.

Unser Modell schließt diese Vorgänge nicht prinzipiell aus, läßt sie aber unberücksichtigt, da die Größenordnung der von ihnen verursachten Änderungen - etwa der „Solarkonstanten “ $\mathrm{S}_{\mathrm{o}}$ - offenbar zu gering ist, um die relativ kurzperiodischen Vorgänge innerhalb des Pleistozäns zu erklären. Darüber hinaus soll unser Modell zeigen, daß auch 
rein terrestrische, also geophysikalische Vorgänge eine ausreichende, quantitative Interpretation der beobachteten Befunde liefern können; hierauf hat auch HoINkEs (1968) vor kurzem hingewiesen. Besonderes Gewicht soll dabei auf die Diskussion des Wärmehaushaltes der Atmosphäre und der Ozeane gelegt werden. Die Anomalien der atmosphärischen Zirkulation lassen sich für eine Eiszeit leicht vorstellen, wenn man sich die fundamentale Rolle der temporären Grenze zwischen Schnee und Eis einerseits, offenem Land und Meer andererseits im Hinblick auf Albedo, Wärmehaushalt und Luftmassenverteilung klar macht.

Unser Modell ist nicht mathematischer Natur; es soll jedoch die physikalischen Parameter eines speziellen paläoklimatischen Modells diskutieren, dessen mathematische Formulierung im Sinne einer theoretischen Klimatologie heute möglich und sinnvoll ist. Hierbei wird man zweckmäßig in mehreren Schritten vorgehen und zuerst Teilprobleme an mehr oder minder vereinfachten Modellen behandeln. Eine solche Entwicklung ist bereits an verschiedenen Orten (Princeton N. J., Leningrad, Tokyo) im Gange, und die Arbeiten von Manabe und Mitarbeitern $(1964,1967)$ haben für wesentliche Teile des PaläoklimaProblems bereits eine quantitativ befriedigende Lösung gegeben.

\section{Empirische Grundlagen}

Versucht man, die für unsere Fragestellung wesentlichen Beobachtungstatsachen aus der Fülle der geologischen Literatur (einschließlich aller Nachbarwissenschaften) herauszufiltern, so ist es notwendig, alle strittigen Fragen auszuklammern und sich auf die Grundtatsachen zu beschränken, die als gesichert angesehen werden. Hierbei stehen im Vordergrund die Monographieen von v. Klebelsberg (1948/49), Woldstedt (19541966), Flint (1957) und Frenzel (1967); hinzu kommt die Darstellung der gesamten Paläoklimatologie durch Schwarzbach (1961). Es ist in diesem Rahmen unmöglich, alle neueren Einzelarbeiten einzeln aufzuführen.

a) Abgesehen von der pleistozänen Vereisung (Dauer insgesamt bisher $\sim 3 \mathrm{Ma}$ oder Megajahre; $1 \mathrm{Ma}=10^{6}$ a) und der permokarbonen Vereisung (insgesamt $\sim 30 \mathrm{Ma}$ ) ist das Klima der voll überschaubaren geologischen Vergangenheit seit dem Beginn des Paläozoikums (Unterkambrium vor rund $550 \mathrm{Ma}$ ) zu etwa $90 \%$ in beiden Polarregionen eisfrei gewesen. Damit ist also der Normalzustand der Erdoberfläche eisfrei oder „akryogen“ - um einen von KERnER-MARILAun (1930) eingeführten Begriff zu gebrauchen. $\mathrm{Da}$ auch vorher im Eokambrium (vor rund $600 \mathrm{Ma}$ ) und in verschiedenen Zeitabschnitten des Präkambriums bis zurück zu etwa $2000 \mathrm{Ma}$ mehr oder minder überzeugende Eiszeitspuren gefunden worden sind, ist offenbar die zweite Hälfte der Erdgeschichte - ohne Zweifel die letzten 10-12\% - durch den Wechsel zwischen langen akryogenen und relativ kurzen kryogenen Zeitabschnitten bezeichnet. Die charakteristische Zeitskala der Warmzeiten liegt bei $10^{8}$ a (Mesozoikum-Alttertiär mindestens $180 \mathrm{Ma}$ ), die der kryogenen Perioden dagegen bei $10^{7}$ a.

b) Im Laufe des Tertiärs kam es in mittleren und höheren Breiten - jedenfalls der Nordhalbkugel - zu einer erst sehr geringen, dann - etwa ab Untermiozän, d. h. vor rund $30 \mathrm{Ma}$ - stärkeren Abkühlung bis zu der Grenze Pliozän/Pleistozän (Abb. 1). Erst von da ab setzen auf den Nordkontinenten die großen Vereisungen und die mit ihnen gekoppelten eustatischen Meeresspiegelschwankungen mit der Zeitskala $10^{4}-10^{5}$ a ein. Diese Abkühlung betraf jedoch nicht (oder nur schwach) die Tropenzone, wo die Oberflächentemperaturen bei $27-28^{\circ} \mathrm{C}$ verblieben. Für die Polargebiete ergibt sich im Alttertiär eine charakteristische Temperatur von $+8-10^{\circ}$, wenn man die Richtung der ozeanischen Tiefen-Zirkulation mit der heutigen gleichsetzt (FLohn 1964) und die Temperatur des tropischen Bodenwassers (Emiliani 1955, 1957, 1961, 1966) mit der des polaren Oberflächenwassers identifiziert. Damals standen also einer bis etwa $50^{\circ}$ Breite reichenden, tropisch-warmen Zone zwei Polarkalotten mit gemäßigtem Klima gegenüber. 


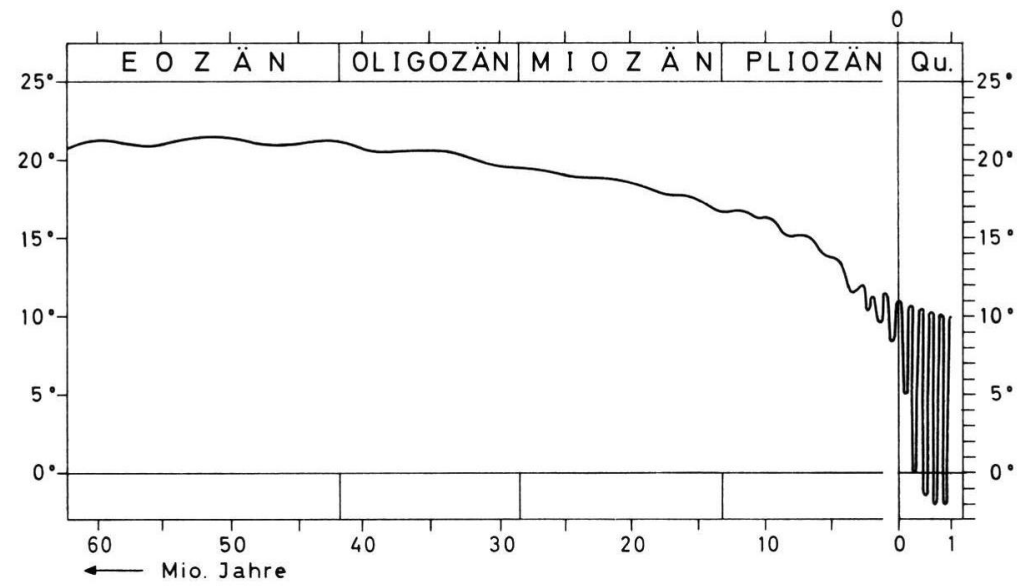

Abb. 1. Abkühlung im Tertiär und Pleistozän (nach Woldstedt).

c) Im Laufe des P li o zäns bildeten sich (vor 2.5-3 Ma) im Bereich von Island, in der Sierra Nevada Kaliforniens und in der Antarktis Gebirgsgletscher; um die gleiche Zeit taucht eisverdriftetes Material in den Bohrkernen der südlichen Ozeane auf (HAYs \& OPDYKE 1967). In der Antarktis könnte schon vor rund $5 \mathrm{Ma}$ (Hoinkes 1968) ein echtes Inlandeis geringer Mächtigkeit existiert haben; hieraus ergibt sich (mit allem Vorbehalt) die Möglichkeit, daß die ersten Gebirgsgletscher in der Antarktika bereits im Altpliozän oder noch früher auftraten.

d) Im Pleistozän kommt es zunächst zur Bildung eines ausgedehnten Inlandeises auf der Antarktis, dessen Dicke zu einem unbekannten Zeitpunkt noch etwa $300 \mathrm{~m}$ größer war als heute. Auf den großen Kontinenten der Nordhalbkugel bildeten sich mehrfach - mindestens 6-8 mal - große Eisschilde von ähnlichem Umfange aus; ihre Bildungszeit läßt sich aus Überlegungen zum Massenhaushalt der Größenordnung nach zu rund 20000 a abschätzen. Ihr Abschmelzen geht etwas rascher vor sich; für die jüngste Eiszeit (Würm bzw. Wisconsin) steht hierfür nur ein Zeitraum von höchstens 13-15000 a zur Verfügung, da zur postglazialen Wärmezeit (vor rund 6000 a) die Inlandeise verschwunden und die Gebirgsgletscher weiter zurückgewichen waren als heute. Antiparallel $\mathrm{zu}$ Bildung und Abbau dieser Eisspeicher auf dem Festland vollzog sich Absinken (maximal auf $-145 \mathrm{~m}$ ) und Wiederanstieg des Meeresspiegels, so daß bis 3.7\% des Wasservorrats der Erde in Form von Eis festgelegt war. In den Warmzeiten stieg der Meeresspiegel um mindestens $15-30 \mathrm{~m}$ gegenüber dem heutigen Niveau an; in verschiedenen Gebieten gibt es Anzeichen für verbreitete Meeresstände bis $+75-80 \mathrm{~m}$, lokal sogar bis $+400 \mathrm{~m}$ und noch höher. Da tektonische Hebungen schwer auszuschließen sind und viele dieser Befunde (Mittelmeer, Westküste Südamerikas) in tektonisch unruhigen Räumen liegen, erscheinen weltweite glazialeustatische Meeresspiegel-Schwankungen (FAIRBRIDGE 1961, 1967, Woldstedt 1966, Flint 1957) oberhalb eines Niveaus $+30 \mathrm{~m}$ offenbar doch noch nicht als völlig gesichert, so daß wir hier von ihrer Diskussion absehen wollen.

e) Dieser in geologischer Sicht kurzperiodische Wechsel zwischen Kalt- und Warmzeiten im Pleistozän mit einer Zeitskala von $10^{4}-10^{5}$ a erfaßte die gesamte Erde außer der Antarktis jeweils gleichzeitig. Dies ist für die jüngste Eiszeit gegen jeden Zweifel gesichert und damit ein eindeutiges Indiz gegen eine primäre Rolle der Erdbahnelemente - diese fordern zwar nicht Gegenläufigkeit der beiden Hemisphären, jedoch einen Zeitunterschied von mindestens $10-11000$ a. Vielfach wird betont, daß es sich hierbei um relativ kurzfristige Umschaltungen zwischen zwei extremen Zuständen handle 
(Flip-Flop-Mechanismus, Vacillation); demgegenüber muß jedoch darauf hingewiesen werden, daß schon aus Massenhaushaltsgründen für den Auf- und Abbau der nordhemisphärischen Eiskalotten Zeiträume von (mindestens) jeweils 15000 a notwendig sind. Ob das grönländische Inlandeis (Fläche $1.7 \cdot 10^{6} \mathrm{~km}^{2}$ ) die Warmzeiten überdauert hat, ist bisher offenbar noch nicht gesichert. Für die Antarktis (Fläche $13 \cdot 10^{6} \mathrm{~km}^{2}$, mittlere Mächtigkeit über $2000 \mathrm{~m}$ ) kann dieses Uberdauern als sicher gelten, zumal Advektion wärmerer Luftmassen zunächst einmal die Akkumulation steigert und der Massenverlust bei den sehr tiefen Temperaturen nicht auf Schmelzen, sondern hauptsächlich auf Abbruch von Eisbergen und Schneefegen zurückzuführen ist. Die Frage nach der genauen Zeitskala muß unter Hinweis auf die miteinander unvereinbaren Auffassungen von Emiliani (1955, 1957, 1961, 1966), Frechen und Lippolt (1965) einerseits, sowie Ericsson und Wollin $(1964,1968)$ andererseits offen bleiben.

f) Die regionale Anordnung der nordhemisphärischen kontinentalen Eisschilde entspricht - jedenfalls für die jüngste Vereisung - nicht den klimatologischen Erwartungen. Nach diesen müßten wir den Beginn der Vereisung bei einer allgemeinen Abkühlung in den heute schon stark vergletscherten Hochgebirgen von Skandinavien, Alaska und den kanadischen Rockies sowie des Karakorum erwarten, in deren Nähe dann

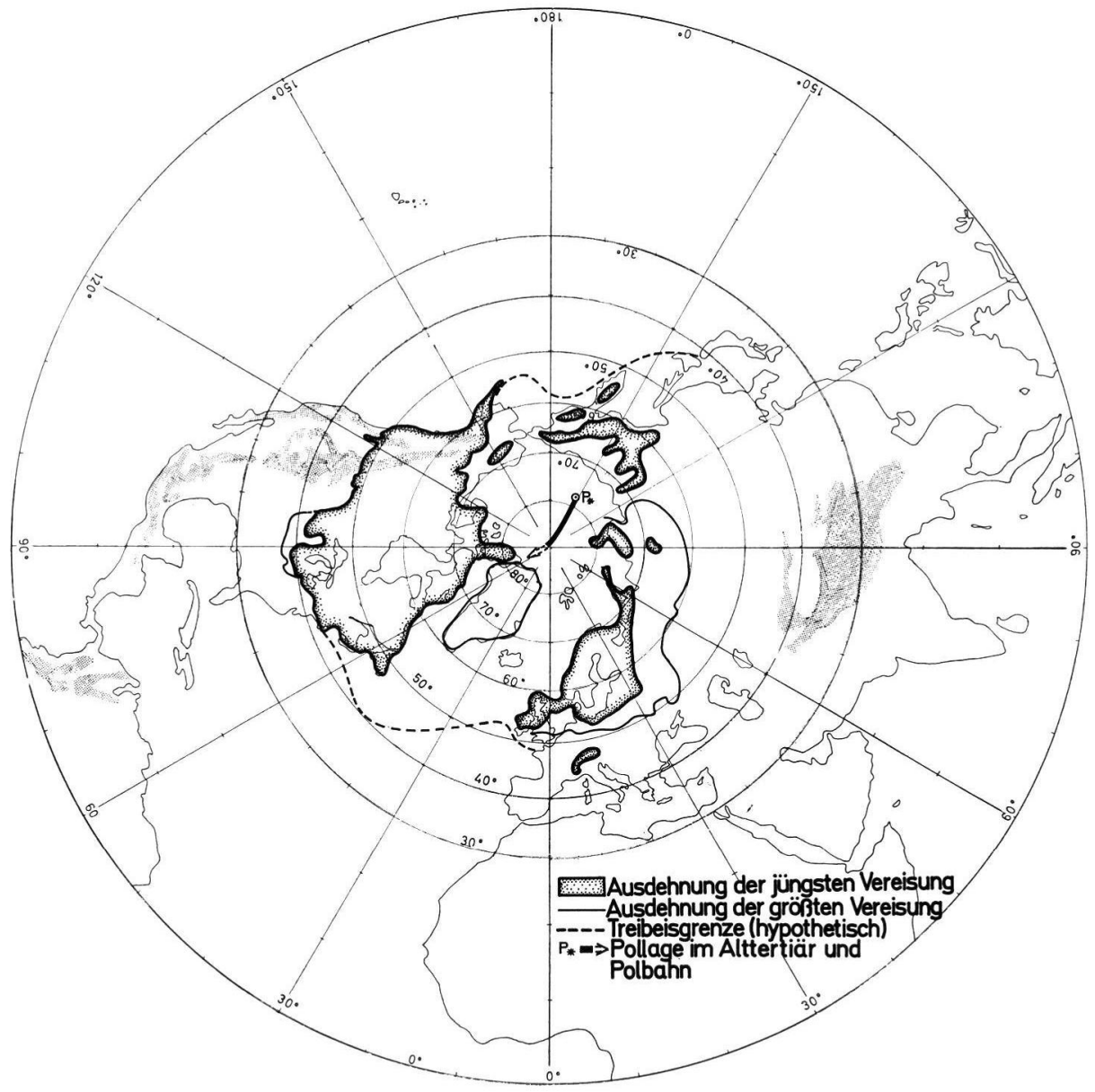

Abb. 2. Vereisung auf der Nordhalbkugel, Treibeisgrenze und Polwanderung. 
auch die Eiszentren gelegen haben müßten. Das gilt aber nur für Skandinavien: die Bildung des größeren nordamerikanischen Inlandeises (Fläche $15 \cdot 10^{6} \mathrm{~km}^{2}$ ) ging offenbar aus von den Gebirgen Nordlabradors (und vielleicht Baffinlands), während sich dann das Zentrum nach dem Keewatin-Flachland westlich der Hudsonbay verlagerte (FLINT 1957); das Kordilleren-Eis war demgegenüber immer unbedeutend. Auch die Vergletscherungen der zentralasiatischen Gebirge blieben in relativ engen Grenzen (v. Wissmann 1959), mit einer Schneegrenzdepression von nicht mehr als 400-700 m (statt $1200-1300 \mathrm{~m}$ in vielen Gebirgen Europas und Nordamerikas). Das heutige Kältezentrum Ostsibiriens war offenbar nur von einer gering mächtigen Firn- und Eisschicht überdeckt; doch gibt es im subtropischen Zentralchina (v. WISSMANN 1937), in Japan (ScHwIND 1967) und in weiteren Gebieten Hoch- und Ostasiens (v. Wissmann 1959) Hinweise auf wesentlich ältere, umfangreiche Gebirgsvereisungen mit einer Schneegrenzdepression von mindestens $2000 \mathrm{~m}$, die $\mathrm{m}$. E. eine sorgfältige und kritische Prüfung mit den neuesten Methoden verdienen.

Eine kartenmäßige Zusammenstellung (Abb. 2) zeigt einen auffälligen Gegensatz zwischen der starken Vereisung des zirkum-atlantischen und der relativ unbedeutenden Vergletscherung des zirkum-pazifischen Sektors, mindestens für die letzte Eiszeit. Dieser Gegensatz erreicht seinen Höhepunkt in dem Vergleich des kaltzeitlichen Küstenklimas der Biskaya (Nordspanien und Westfrankreich) einerseits - nach KaISER (1967) mit einer ganzjährigen Abkühlung von mindestens $12^{\circ}$, wahrscheinlich $15^{\circ} \mathrm{C}$ - und der sogar etwas nördlicher gelegenen Olympus-Halbinsel an der amerikanischen Pazifikküste nach Heusser (1966) mit einer kaltzeitlichen Abkühlung von nicht mehr als $5-6^{\circ} \mathrm{C}$ (genau so in Südchile). Da beide Gebiete für maritimes Westwindklima repräsentativ sind, in weitem Abstand von den großen Inlandeisgebieten, erscheint eine rein lokale Interpretation hier kaum möglich. Die kontinentale Vereisung Nordamerikas erreichte in der vorletzten Eiszeit $38^{\circ}$ Breite, diejenige Europas in Mitteleuropa $51^{\circ}$, im Don-Gebiet $48^{\circ} \mathrm{N}$; die Asymmetrie zum geographischen Nordpol ist evident. Ein hypothetischer Vereisungspol käme etwa nach Grönland $\left(75^{\circ} \mathrm{N}, 40^{\circ} \mathrm{W}\right)$ zu liegen; auf die eventuelle Rolle einer Polwanderung gehen wir später ein (Kap. 3 a).

g) In den Trope n verfügen wir außer den oft zitierten Isotopen-Temperaturmessungen von EmiLiani $(1955,1957,1961,1966)$ über glaziologische und palynologische Befunde aus den Hochländern; sie belegen übereinstimmend eine mehrmalige kaltzeitliche Abkühlung um 5-6 $6^{\circ}$, im Extremfall vielleicht lokal (im nördlichen Südamerika) um $8^{\circ}$. Letzterer Wert darf aber kaum als repräsentativ angesehen werden, da eine Abkühlung der tropischen Ozeane von $27^{\circ}$ auf $19^{\circ}$ wohl kaum die charakteristischen Korallen am Leben erhalten hätte. Hierbei herrscht aber offenbar doch eine praktisch vollständige $\mathrm{Pa}$ rallelität mit den thermischen Fluktuationen der ganzen Erde (außer der Antarktis, die stets eine Sonderstellung einnimmt). In diesem Fall kann die von Bernard (1962) vorgeschlagene Interpretation der Erdbahnelemente mit ihrer sehr kurzen Zeitskala (21 $000 \mathrm{a})$ nicht herangezogen werden, die auch nur für die jahreszeitliche Verteilung der tropischäquatorialen Temperaturen und Regenzeiten gilt; selbst in der von EmILIANI angenommenen kurzen Zeitskala müßten etwa 20 derartige Schwankungen aufgetreten sein.

\section{Geophysikalische Hypothesen}

Im Gegensatz zu früheren Jahrzehnten haben sich in den letzten 15 Jahren - besonders seit der kritischen Anwendung paläomagnetischer Methoden - so viele in sich nahezu widerspruchsfreie Daten über die Veränderung des Gradnetzes der Erde angesammelt, daß die viel diskutierten Probleme der Verlagerung der Rotationspole und der gegenseitigen Verschiebung der Kontinentalschollen heute prinzipiell gesichert sind; die Diskussion geht nur mehr um die Fragen nach der Zeit, Geschwindigkeit und Richtung der Verlagerung. Für unsere Fragestellung können wir uns auf die wichtigsten Befunde beschränken (Cox 1968, RunCORN u. a. 1965). 
a) Die paläomagnetischen Daten für die Pollagen aus den verschiedenen Kontinentalschollen sind nur dann widerspruchsfrei miteinander zu vereinbaren, wenn wir Relativbewegungen der Kontinente zulassen. Im Alttertiär (vor 40-50 Ma) lag der (magnetisch ermittelte) Nordpol nördlich der Nordsibirischen Inseln (Cox 1968) etwa in $78^{\circ} \mathrm{N}$, $153^{\circ} \mathrm{E}$ (Abb. 2), seine Wanderungsgeschwindigkeit seit dieser Zeit ergibt sich im Mittel auf etwa $3 \mathrm{~cm} / \mathrm{a}$. Damit stimmt das Ergebnis der seit 70 Jahren durchgeführten astronomischen Polhöhen-Bestimmungen von 6 der Länge nach gut verteilten Sternwarten in $39.1^{\circ}$ N-Breite erstaunlich gut überein: nach Mаякоwiтz, A. Sтоуко u. a. (1968) beträgt das säkulare Glied der Polve r la ge r u n - befreit von allen kurzperiodischen Schwankungen - im Mittel etwa $10 \mathrm{~cm} / \mathrm{a}\left(\sim 1^{\circ} / \mathrm{Ma}\right)$ in der Richtung nach Labrador $\left(65^{\circ} \mathrm{W}\right)$. Damit ist für das Pleistozän (und jüngste Pliozän) gesichert, daß die Lage der Rotationspole nicht mehr als 1-3 Breitengrade von der heutigen Lage abweicht; in dem Ursachenkomplex der pleistozänen Eiszeiten spielt dann irgendeine Polverlagerung nur eine untergeordnete Rolle.

b) Eine zahlenmäßige Interpretation der paläomagnetischen Daten für die Kontinente stößt mangels eines verbindlichen und zeitlich konstanten Bezugssystems auf Schwierigkeiten. Inzwischen hat sich aber auf den Ozeanböden eine neue Datenquelle (VINE 1966, Pitman 1968) ergeben: hier bilden sich die in unregelmäßigen Abständen - Zeitskala $10^{5}-10^{6}$ a - erfolgenden Umkehrungen des erdmagnetischen Feldes in Form langgestreckter Streifen entgegengesetzter Magnetisierung parallel zu den großen ozeanischen Schwellen ab. In diesen Schwellen - wie im zentralen Atlantik oder im Indik - existieren große Grabenbrüche mit auffällig starkem Relief, in denen der lokal um einen Faktor 3-8 vergrößerte Wärmestrom aus dem Erdinnern die Existenz aufwärts steigender heißer Magmen anzeigt (Abb. 3). Von diesen zentralen Gräben - nicht identisch mit den randlichen Tiefseegräben der Ozeane! - aus erfolgt nach einer von S. Dietz und Hess vorgeschlagenen "S preizungstheorie der Ozea ne" eine seitliche Ausbreitung des aufgequollenen Magmas, das die zur Zeit der Abkühlung unter den Curie-Punkt herrschende magnetische Feldrichtung konserviert. Diese seitliche Ausbreitung erfolgt mit Geschwindigkeiten von $1-5 \mathrm{~cm} / \mathrm{a}$, die mittels radioaktiver IsotopenMethoden bestimmt worden sind; sie läßt sich in einigen Gebieten - unter der Voraussetzung konstanter Spreizraten - bis in die Kreidezeit (vor $75 \mathrm{Ma}$ ) zurückverfolgen

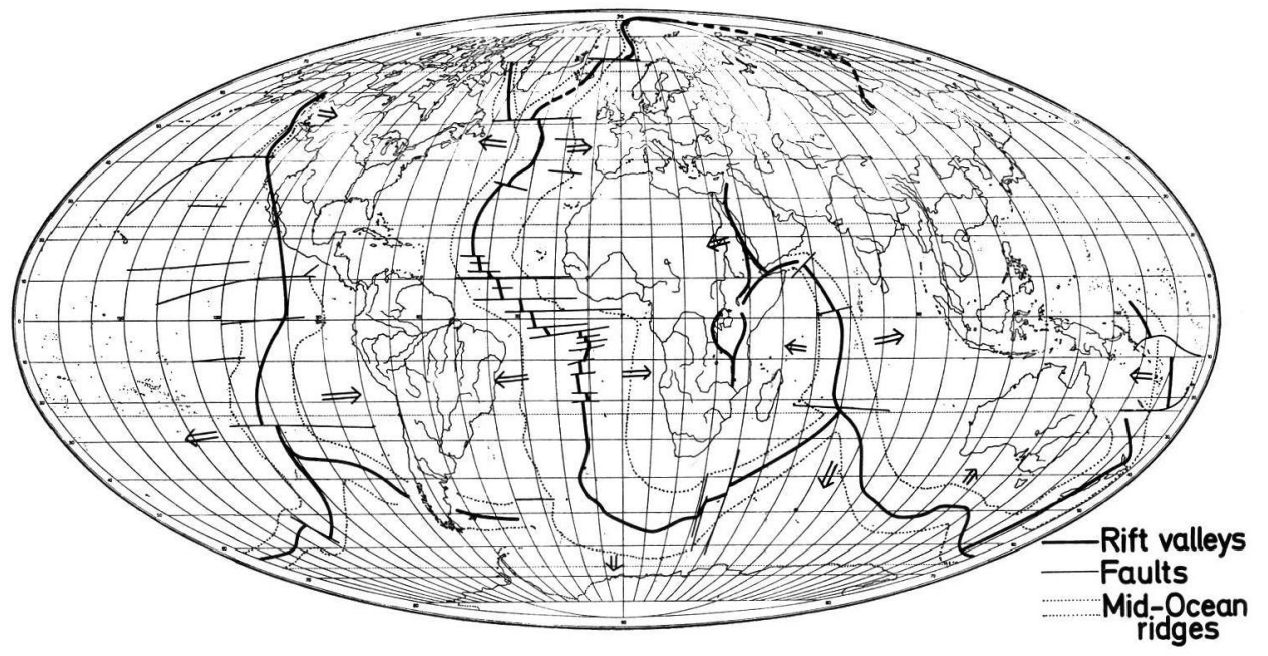

Abb. 3. Spreizung der Ozeanböden und Kontinentaldrift (nach HeEzen und Tharp). 
(Pitman 1968). Damit ist die Theorie stationärer Konvektionszellen (RunCORN 1965) im Erdmantel - die für Fragen der Großtektonik und Gebirgsbildung schon öfters diskutiert worden ist - im Prinzip bestätigt; den Aufquellzonen in den Ozeanen mit divergierender Strömung entsprechen umgekehrt Verschluckungs- und Pressungszonen unter den Kontinenten mit konvergierender Strömung. (Diese Interpretation liefert also kein Argument für die Frage nach einer Expansion der Erde oder für eine Änderung der Gravitationskonstanten).

Für unsere Fragestellung ergibt sich hieraus zunächst, daß der a n ta $\mathrm{kt}$ is che $\mathrm{K}$ o $\mathrm{n} \mathrm{t}$ in e $\mathrm{n}$ ein Teil des riesigen paläozoischen Gondwana-Kontinents war, der aus Südamerika, Afrika, Madagaskar, der indischen Dekkan-Scholle sowie aus Australien und Antarktika bestand. Dieser Kontinent lag im Perm und Oberkarbon etwa zentral zum Südpol; seine Aufspaltung und radiale Drift begann etwa an der Wende zwischen Perm und Mesozoikum, die Bildung des Südatlantiks erst zu Beginn der Kreide. Die heutige antarktische Scholle driftete aus dem Bereich des Indischen Ozeans nach Süden; die rezente Spreizgeschwindigkeit des Ozeanbodens liefert eine rasche Drift von etwa $6 \mathrm{~cm} / \mathrm{a}$ (Pitman 1968). Nehmen wir diese Rate als konstant an, dann wäre die antarktische Scholle vor etwa $60 \mathrm{Ma}$ mit ihrer Vorderseite (im ältesten Tertiär) in Polnähe gerückt und hätte vor etwa $35 \mathrm{Ma}$ (im oberen Oligozän) den Pol selbst erreicht, wobei die oben erörterten Polverlagerungen vernachlässigt wurden; diese könnten die Zeitskala um einen Faktor $\sim 2$ beschleunigt haben.

c) Die Glaziologi e als geophysikalische Disziplin befaßt sich mit der Mechanik der Gletscherbewegung sowie mit dem Massen- und Wärmehaushalt der Gletscher. Hierbei hat sich (HoInkes 1961) ein fundamentaler Unterschied zwischen kalten Gletschern - deren Temperatur überall unter dem Schmelzpunkt liegt - und warmen Gletschern ergeben. Die ersteren (Typ Antarktis) bewegen sich nur langsam an Scherflächen, mit geringer Beteiligung plastischer Vorgänge; wegen der hohen Albedo (0.8-0.9) ihrer Oberfläche bleibt die sommerliche Ablation und der Massenumsatz sehr gering. Demgegenüber läuft die Bewegung der warmen Gletscher (Typ Alpen) als Folge der Schmelzungs- und Regelationsvorgänge sowie der dann entstehenden Reibungswärme im Gletscherkörper wesentlich rascher ab; wegen der niedrigen Albedo in der Schmelzperiode (0.3-0.4) sind Ablation und Massenumsatz wesentlich größer. Das Studium der früher rätselhaften raschen Gletscherausbrüche (Surges) in hohen Breiten hat gezeigt, daß in einem kalten Gletscher durch fortschreitende Akkumulation nach Erreichen des druckabhängigen Schmelzpunktes plötzlich ein Übergang zu einem warmen Gletscher erfolgt, wobei dann ein Teil der Gletschermasse plastisch mit hohen Geschwindigkeiten (100 m pro Tag und mehr) ausfließen kann.

Aus diesen Befunden hat der neuseeländische Geophysiker A. T. WILSON (1964) eine neue, recht attraktive Eiszeit-Theorie (WILsON 1964, 1966) entwickelt. Sie geht aus von einem langsam anwachsenden Eisschild der Antarktis (heutige Massenbilanz sehr wahrscheinlich mit $+2-3 \mathrm{~cm} /$ a positiv): nachdem der Druckschmelzpunkt am Boden einmal in großen Gebieten überschritten ist, kommt es zu einer allseitigen raschen und weiten Ausbreitung eines Schelfeises vom Typ des Roß-Eisschelfes mit seiner mittleren Dicke von $300 \mathrm{~m}$. Wilson nimmt eine derartige Ausbreitung bis in die Höhe der ozeanischen antarktischen Konvergenz an, wo in einer mittleren Breite von $55^{\circ} \mathrm{S}$ das kalte antarktische Wasser $\left(+2\right.$ bis $\left.+4^{\circ} \mathrm{C}\right)$ unter das etwa $5^{\circ}$ wärmere subpolare Wasser absinkt. In der Tat findet sich in diesem Bereich am Meeresboden eisverdriftetes glaziomarines Material mit einem Alter von mehreren Ma (Hays \& Opdyke 1967). Auf die Folgen eines derartigen Vorstoßes für den Wärmehaushalt der Erde gehen wir in Abschnitt 4 näher ein. Diese Theorie hat besondere Aktualität erlangt, seit eine Tiefbohrung in der Antarktis nahe der Byrd-Station $\left(80^{\circ} \mathrm{S}, 120^{\circ} \mathrm{W}\right)$ am Grund des Bohrloches in $2164 \mathrm{~m}$ Tiefe unter starkem 
Druck stehendes Schmelzwasser beim Druckschmelzpunkt von $-1.6^{\circ}$ antraf (Gow 1968). Dieser Befund steht im Gegensatz zu Grönland, wo die Bodentemperatur unterhalb des Druckschmelzpunktes blieb; die weitere Diskussion verspricht äußerst interessant zu werden.

d) Auch von oze a nographis cher Seite hat das Eiszeitproblem neue Aspekte erhalten; diese werfen ein neues Licht auf die (unter $2 \mathrm{f}$ ) erörterten regionalen Unterschiede zwischen dem pazifischen und dem atlantischen Sektor. So geht WEYL (1968) in seinen Überlegungen davon aus, daß die Meereisbildung in einem stabil geschichteten Ozean mit einer salzarmen Oberschicht viel rascher - d. h. nach geringerem Wärmeentzug - vor sich geht als in einem gut durchmischten Ozean mit hohem Salzgehalt. Heute beläuft sich der Salzgehalt des Atlantiks auf rund 37\% gegenüber nur $35-36 \%$ im Pazifik. Um nun die Einleitung der relativ viel stärkeren Vereisung im Atlantik-Sektor zu erklären, postuliert WEYL eine entscheidende Schwächung der tropischen Ostwinde, die heute einen wesentlichen Teil des atmosphärischen Wasserdampfes über Mittelamerika hinweg in den Pazifik verfrachten; damit soll sich die Salzgehaltdifferenz Atlantik-Pazifik umkehren und eine stärkere Vereisung des Nordatlantik verursacht werden. Nun ist eine solche Zirkulationsanomalie zwar als Folge der Ausbildung des nordamerikanischen Inlandeises gut vorstellbar, aber nicht als dessen Voraussetzung (vgl. Kapitel 5 d).

Zweifellos spielen aber die heute als grundlegend erkannten Wechselwirkungen zwischen Atmosphäre und Ozean auch für das Eiszeitproblem eine Rolle. In diesem Rahmen mußte im Atlantik die große Menge der sommerlichen Schmelzwasser der beiden kontinentalen Eisschilde ähnlich wie heute im arktischen Ozean zur Bildung einer ausgedehnten flachen salzarmen Oberschicht beitragen, die den salzreichen warmen Golfstrom schon in mittleren Breiten (vielleicht $\mathrm{ab} 50^{\circ} \mathrm{N}$ ) überdeckt und damit seine Heizwirkung für die europäischen Westküsten weitgehend ausschaltet. Mit der ganzjährigen Verlagerung der amerikanisch-atlantischen Hauptfrontalzone nach $35-40^{\circ} \mathrm{N}$ (am Südrand des amerikanischen Inlandeises) wird auch der Hauptstrich des Golfstromes eher südlicher ansetzen als heute, und an den europäischen Küsten kann ein größerer Teil in den Kanarenstrom nach $\mathrm{S}$ abgelenkt werden. Wenn dann die a $\mathrm{t} \mathrm{l}$ a $\mathrm{t}$ is che Treibeisgrenze etwa bis zu einer Linie Neufundland-Irland nach $S$ vorrückt, kann ein Teil dieses Treibeises auch in die Biskaya gelangen und dort besonders im Sommer die oben (unter $2 \mathrm{f}$ ) geschilderte exzessive Temperaturdepression (mit subarktischen Sommertemperaturen von 8$10^{\circ} \mathrm{C}$ !) hervorrufen, die wegen der großen Entfernung zum Eisrand (am Niederrhein bzw. Genfer See) anders kaum verständlich sind. An allen Pazifikküsten sind die Schmelzwasserzufuhren gering; daher fehlt dort die salzarme Deckschicht, und die eiszeitliche Temperaturdepression ist überall genau so groß wie in der Äquatorzone.

\section{Fragen des Strahlungs- und Wärmehaushaltes}

a) Die Diskussion des Strahlungs- und Wärmehaushaltes für alle paläoklimatischen Probleme muß ausgehen von der fundamentalen Gleichung der „extraterrestrischen“ Strahlungsbilanz, d. h. des Strahlungsgleichgewichtes an der Obergrenze der Atmosphäre (durch den Index o bezeichnet):

$$
\mathrm{S}_{\mathrm{o}}\left(1-\mathrm{a}_{\mathrm{p}}\right) \pi \mathrm{r}^{2}=4 \pi \mathrm{r}^{2} \mathrm{E}_{\mathrm{o}}=4 \pi \mathrm{r}^{2} \varepsilon \sigma \mathrm{T}_{\mathrm{R}}{ }^{4}
$$

Hierin bezeichnet $\mathrm{S}_{\mathrm{o}}$ die Zustrahlung durch die Sonne - die sogenannte "Solarkonstante“ - bezogen auf eine zu ihr senkrechte Fläche, $\mathrm{r}$ den Erdradius, $\mathrm{E}_{\mathrm{o}}=\varepsilon \cdot \sigma \mathrm{T}_{\mathrm{R}}{ }^{4}$ die terrestrische Ausstrahlung, $a_{p}$ die planetarische Albedo und $\varepsilon$ das (langwellige) Emissionsvermögen des Systems Erde und Atmosphäre, $\sigma$ die Stefan-Boltzmann'sche Konstante und $\mathrm{T}_{\mathrm{R}}$ die 
effektive Strahlungstemperatur (in ${ }^{\circ} \mathrm{K}$ ) des gleichen Systems. Unter der - üblichen, aber nicht ganz berechtigten - Annahme $\varepsilon=1$ ergibt sich die effektive Temperatur eines schwarz strahlenden Körpers zu

$$
\mathrm{T}_{\mathrm{R}}{ }^{4}=\mathrm{S}_{\mathrm{o}}\left(1-\mathrm{a}_{\mathrm{p}}\right) / 4 \sigma
$$

Die Zahlenwerte dieses Schwarzstrahlungsmodells sind in Abb. 4 dargestellt; als Einheit für die Strahlungsmenge pro Flächeneinheit gilt 1 Langley $(\mathrm{Ly})=\mathrm{gcal} \mathrm{cm}^{-2}$. Für die heute meist angenommenen Werte von $S_{o}=2 \mathrm{Ly} / \mathrm{min}$ und $\mathrm{a}_{\mathrm{p}}=0.35$ ergibt sich $\mathrm{T}_{\mathrm{R}}=$ $250^{\circ} \mathrm{K}$ oder $-23^{\circ} \mathrm{C}$, etwa entsprechend einer mittleren Höhe der atmosphärischen Emissionsschicht von rund $6 \mathrm{~km}$ oder $450 \mathrm{mb}$. Die neueste Auswertung der Strahlungsmessungen von Satelliten (RAschKe 1968) liefert andere Werte: $\mathrm{T}_{\mathrm{R}}=255^{\circ} \mathrm{K}, \mathrm{a}_{\mathrm{p}}=0.30$. Diese Werte stehen im Gleichgewicht mit $\mathrm{S}_{0} \sim 1.96 \mathrm{Ly} / \mathrm{min}$, was wiederum gut zu den neuesten Bestimmungen von $\mathrm{S}_{\mathrm{o}}=1.95 \mathrm{Ly} / \mathrm{min}$ im Stratosphärenflugzeug (LANE \& DrUmmond 1968) paßt. Für paläoklimatische Zwecke lassen sich aus Abb. 4 beliebige Wertetripel von $\mathrm{S}_{\mathrm{o}}, \mathrm{T}_{\mathrm{R}}$ und $\mathrm{a}_{\mathrm{p}}$ näherungsweise abschätzen.

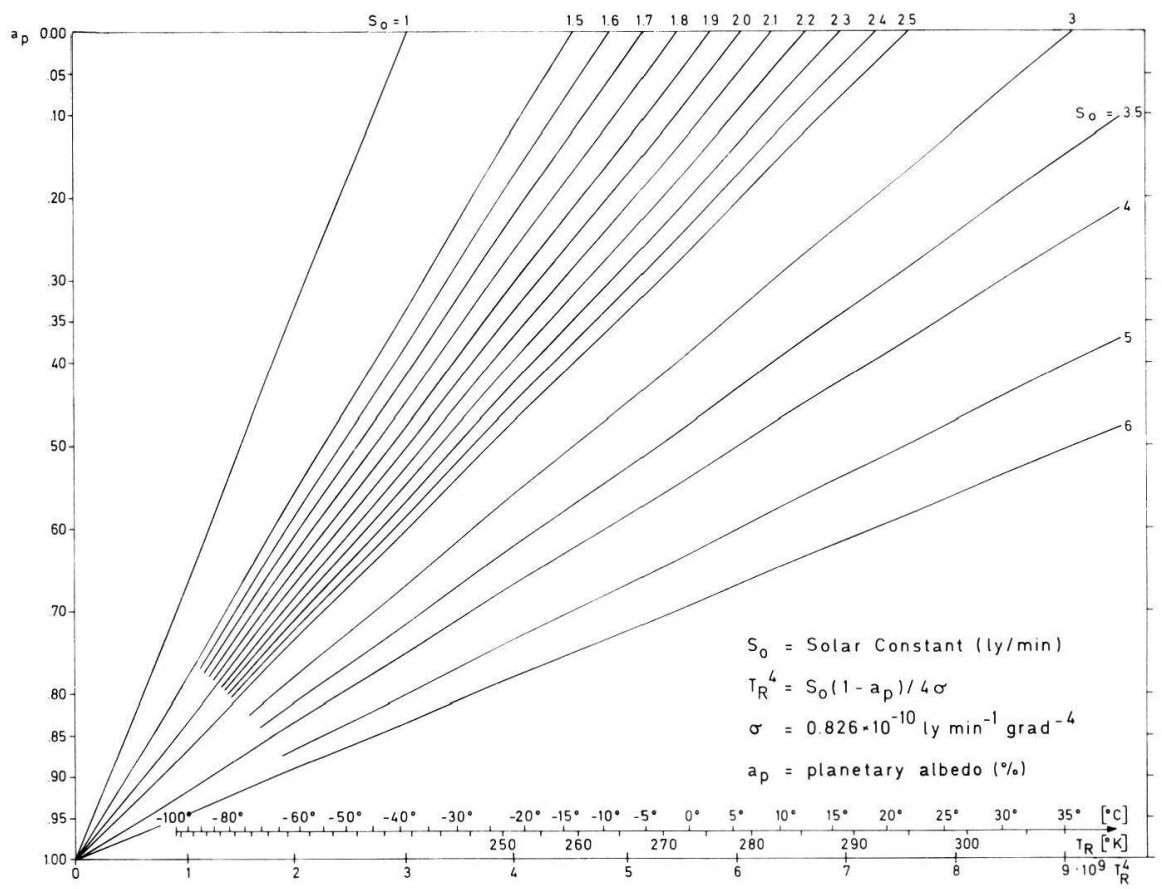

Abb. 4. Strahlungstemperatur des Planeten Erde in Abhängigkeit von Solarkonstante und planetarischer Albedo.

b) In grober Näherung kann man versuchen, die Änderung der für uns wichtigeren Größe der mittleren Luftemperatur in $2 \mathrm{~m}$ Höhe $\mathrm{T}_{\mathrm{S}}$ abzuschätzen aus

$$
\frac{\mathrm{dT}_{\mathrm{R}}}{\mathrm{T}_{\mathrm{R}}}=\frac{\mathrm{dT}_{\mathrm{S}}}{\mathrm{T}_{\mathrm{S}}}
$$

Die wirkliche Beziehung zwischen $\mathrm{T}_{\mathrm{R}}$ und $\mathrm{T}_{\mathrm{S}}$ ist viel komplexer; Rechnungen mit dem Strahlungsmodell von Manabe $(1964,1967)$ liefern wesentlich realistischere Werte für $\mathrm{T}_{\mathrm{S}}$ als Funktion von $\mathrm{S}_{\mathrm{o}}$ und der Albedo der Erdoberfläche $\mathrm{a}_{\mathrm{s}}$ (Abb. 5). Für eine Änderung der Solarkonstante $S_{o}$ um 1\% ergibt sich in dem Bereich nahe 2 Ly/min bei dem Schwarz- 


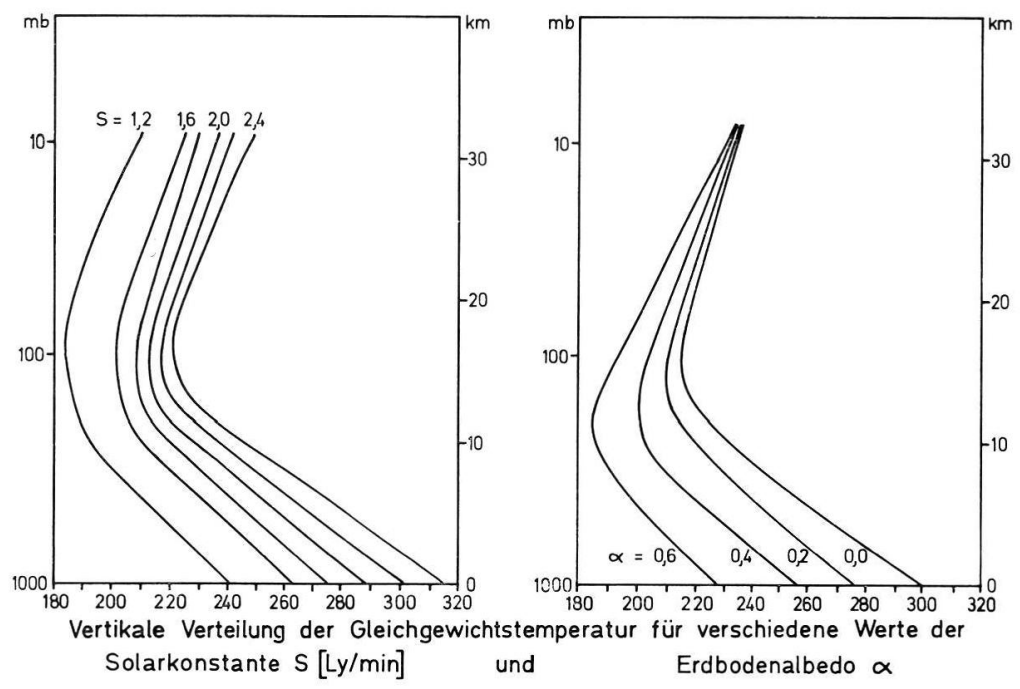

Abb. 5. Temperaturschichtung in Abhängigkeit von Solarkonstante und Oberflächenalbedo (nach MANABE)

strahlungsmodell eine gleichsinnige Änderung von $\mathrm{T}_{R}$ um $0.67^{\circ} \mathrm{C}$, dagegen nach ManabE (1967) (bei konstanter relativer Feuchte) eine Anderung von $\mathrm{T}_{\mathrm{S}}$ um $1.30^{\circ} \mathrm{C}$. Eine globale Abkühlung $\mathrm{dT}_{\mathrm{s}}$ in den Kaltzeiten um $5^{\circ} \mathrm{C}$ ist also (bei $\mathrm{a}_{\mathrm{p}}=$ const.) äquivalent einem Absinken von $S_{0}$ von 1.95 auf 1.87 .

Diese Ableitung ist aber nur von hypothetischem Interesse, denn mit der Bildung der kontinentalen Eisfelder ändert sich auch die mittlere A $1 \mathrm{bed}$ o $\mathrm{a}_{5}$ und $\mathrm{a}_{\mathrm{p}}$; diesen schon von Wundt $(1938,1939,1944)$ betonten Effekt hat Milankovitch $(1930,1938)$ in seinen neueren Rechnungen (1938) berücksichtigt. Nehmen wir in einer Kaltzeit eine Zunahme der Schneefläche auf den Kontinenten um $30.10^{6} \mathrm{~km}^{2}$ an $\left(\right.$ mit $\mathrm{a}_{\mathrm{s}}=0.70$ ), eine Zunahme der Treibeisfläche um ebenfalls $30 \cdot 10^{6} \mathrm{~km}^{2}$ (mit $\mathrm{a}_{\mathrm{s}}=0.40$ ), dann ergibt sich eine Zunahme des heutigen Wertes $a_{s}=0.103$ (Land $\sim 0.18$, See 0.075 ) auf 0.156. Nach MAnABE (1967) entspricht einer Zunahme von $\mathrm{a}_{\mathrm{s}}$ um 0.01 (wieder bei konstanter relativer Feuchte) eine Abnahme von $\mathrm{T}_{\mathrm{s}}$ um $1.2^{\circ} \mathrm{C}$; für obige Anderung von $\mathrm{a}_{\mathrm{s}}$ ergibt sich also eine Abkühlung um 6.4 . Die beobachtete Abnahme der Oberflächentemperatur steht also im BilanzGleichgewicht mit einer realistischen Abschätzung der Albedo-Änderung als Folge der Vereisung; der beobachtete stationäre Zustand während einer Kaltzeit ist also auch bei völliger Konstanz von $\mathrm{S}_{\mathrm{o}}$ möglich.

Gehen wir von der unter 3 c) erwähnten Hypothese von WiLson $(1964,1966)$ aus, so lieferte ein Ausbruch des Antarktik-Eises als Schelfeis bis zu einer mittleren Breite von $55^{\circ} \mathrm{S}$ eine Vergrößerung der Eisfläche von rund 15 auf $46 \cdot 10^{6} \mathrm{~km}^{2}$, wobei wir der Einfachheit halber die Fläche des antarktischen Treibeises während des Ausbruches als konstant annehmen. Damit wächst für $31 \cdot 10^{6} \mathrm{~km}^{2} a_{\mathrm{s}}$ von 0.075 auf 0.80 ; die mittlere Albedo $a_{s}$ der Erdoberfläche ergibt sich dann für die Südhalbkugel zu etwa 0.20, was einem Temperaturrückgang um $12^{\circ}$ entspricht. WiLson's Hypothese reicht also in der Tat aus, eine globale Abkühlung der Mitteltemperatur der Troposphäre um $5-6^{\circ}$ zu erzielen, die sich (bei einer mittleren Verweilzeit der Luft in einer Hemisphäre von 1-2 a) in relativ sehr kurzer Zeit nicht nur über die Südhalbkugel, sondern durch die Barriere schwächeren Austauschs in der Tropenzone hinweg auch (abgeschwächt) über die Nordhalbkugel ausbreiten müßte. 
Das Strahlungsmodell von Manabe $(1964,1967)$ liefert $T_{\mathrm{s}}$ auch für alle möglichen Variationen des Anteils der absorbierenden $\mathrm{Gase} \mathrm{H}_{2} \mathrm{O}, \mathrm{CO}_{2}$ und $\mathrm{O}_{3}$; von diesen dürfen wir $\mathrm{CO}_{2}$ und $\mathrm{O}_{3}$ während des Pleistozäns als nahezu konstant betrachten. Auf die Rolle des Wasserdampfes kommen wir noch (unter $4 \mathrm{~d}$ ) zurück.

c) Die mehrfache pleistozäne Abkühlung der Oberflächente m peratur der tropischen Ozeane- deren Belege allerdings bisher ausschließlich vom Atlantik stammen - wird vielfach als Beleg für eine primäre Änderung von $S_{0}$ interpretiert. Doch hat bereits WUNDT $(1938,1939,1944)$ die Möglichkeit einer sekundären, advektiv von den vereisten Nordkontinenten her induzierten Abkühlung hervorgehoben. Ein solcher Sekundäreffekt erscheint zunächst als wenig wahrscheinlich, da die Mitteltemperatur der tropischen Meeresfläche mit $27-28^{\circ} \mathrm{C}$ heute - bei einer Vereisung von insgesamt $42 \cdot 10^{6} \mathrm{~km}^{2}$ (Land 16, Meer 26) oder $8 \%$ der Erdoberfläche -, im älteren Tertiär $(0 \%)$ und in den pleistozänen Warmzeiten nahezu dieselbe war, während sie in den pleistozänen Kaltzeiten (Vereisung Land 49, Meer mindestens 33, insgesamt $82 \cdot 10^{6} \mathrm{~km}^{2}$ oder $16 \%$ ) jeweils auf $21-22^{\circ}$ absank.

Diese qualitative Betrachtung muß jedoch durch eine $\mathrm{zahlenmäßige} \mathrm{Ab-}$ schätzung der Wärmehaushaltsgrößen der tropischen Ozeane überprüft werden. Da heute - nach Budyko (1963) und Sellers (1965) - in Nähe des Aquators der meridionale Wärmetransport $\mathrm{W}_{\mathrm{T}}$ durch Luft und Ozean verschwindet, beschränken wir uns auf die Zone $0-20^{\circ} \mathrm{N}$, mit einem Landanteil von $22 \%$ und einer Ozeanfläche von rund $70 \cdot 10^{6} \mathrm{~km}^{2}=7 \cdot 10^{17} \mathrm{~cm}^{2}$. Die Gleichung für den Wärmehaushalt des Ozeans können wir schreiben

$$
\mathrm{Q}_{\mathrm{M}}=\mathrm{U}_{\mathrm{L}}+\mathrm{U}_{\mathrm{V}}+\operatorname{div} \mathrm{W}_{\mathrm{T}}+\Delta \mathrm{W}_{\mathrm{M}}
$$

Hierbei bedeuten $\mathrm{Q}_{\mathrm{M}}$ die Strahlungsbilanz (net radiation) an der Meeresoberfläche, $\mathrm{U}_{\mathrm{L}}$ bzw. $U_{V}$ die vertikalen (turbulenten) Umsätze fühlbarer bzw. latenter Wärme (Verdunstung) zwischen Meer und Atmosphäre, div $W_{T}$ die Divergenz des Wärmetransportes im Meer $\left(W_{T M}\right)$ und $\Delta W_{M}$ die Wärmespeicherung im Meer. Die sehr kleine Wärmespeicherung in der Atmosphäre kann vernachlässigt werden, ebenso vernachlässigen wir die Divergenz des Wärmetransportes in der Luft ( $W_{\mathrm{TL}}$, siehe unten). In unserer Betrachtung wird davon abgesehen, div $W_{T}$ noch zu unterteilen; der heutige Unterschied mag als repräsentativ gelten.

Die heutigen Werte für diese Zone (Budyko 1963, Sellers 1965) sind (in Langley pro Tag) $\mathrm{Q}_{\mathrm{M}}=320, \mathrm{U}_{\mathrm{L}}=14, \mathrm{U}_{\mathrm{V}}=276 \mathrm{Ly} / \mathrm{d}$; Tabelle $1 \mathrm{gibt}$ diese Werte nach Umrechnung auf das Jahr und die Fläche $7 \cdot 1017 \mathrm{~cm}^{2}$. Wenn wir Klimaschwankungen vernachlässigen und $\Delta \mathrm{W}_{\mathrm{M}}=0$ setzen, dann bleibt heute für die Wärmetransporte über $20^{\circ} \mathrm{N}$ nach $\mathrm{N}$ noch $1.55 \cdot 1022 \mathrm{cal} / \mathrm{a}$ oder $19 \%$ von $\mathrm{Q}_{\mathrm{M}}$ übrig; davon transportieren $\mathrm{W}_{\mathrm{TM}} 1.2$ und $\mathrm{W}_{\mathrm{TL}}$ $0.35 \cdot 10^{22} \mathrm{cal} / \mathrm{a}$. Diese Betrachtung bezieht sich nur auf den Meeresanteil; der Transport fühlbarer Wärme über Land $\left(\mathrm{W}_{\mathrm{TL}}\right)$ - der mit etwa $1.7 \cdot 10^{22} \mathrm{cal} / \mathrm{a}$ wesentlich größer ist als der über dem Meer - wird als konstant angesetzt. Unsere Voraussetzung $\Delta \mathrm{W}_{\mathrm{XI}}=0$ ist berechtigt: die rezente Klimaschwankung lieferte in der ersten Hälfte des 20. Jahrhunderts eine Erwärmung des Ozeanwassers um $0.01^{\circ} \mathrm{C} / \mathrm{a}$. Nehmen wir eine Speicherung nur innerhalb der etwa $100 \mathrm{~m}$ mächtigen warmen Oberschicht (Troposphäre) des Ozeans (oberhalb der Thermokline) an, so verbraucht diese $7 \cdot 1019 \mathrm{cal} / \mathrm{a}$ oder weniger als $1 \%$ von $Q_{M}$. Die postglazialen und pleistozänen Temperaturänderungen von $6^{\circ}$ in einer Zeitspanne von $10^{3}-10^{4}$ a sind von gleicher Größenordnung; die möglichen Änderungen der Wärmehaushaltsgrößen sind viel zu groß, um aus ihnen auch nur das Vorzeichen einer Klimaänderung abschätzen zu können. Wir betrachten mehrere Modellfälle: 
Tabelle 1

Wärmehaushaltsgrößen für den tropischen $\mathrm{Ozean}\left(0-20^{\circ} \mathrm{N}\right)$

\begin{tabular}{|c|c|c|c|c|c|c|c|c|}
\hline \multirow{4}{*}{ heute } & \multirow{2}{*}{$\mathrm{UV}_{\mathrm{V}}$} & \multirow{2}{*}{$\mathrm{U}_{\mathrm{L}}$} & \multirow{2}{*}{$\operatorname{div} \mathbb{W}_{\mathrm{T}}$} & \multirow{2}{*}{$Q_{M}$} & \multicolumn{4}{|c|}{ Anderungen gegenüber heute (in $\% / 0$ ) } \\
\hline & & & & & $\mathrm{U}_{\mathrm{V}}$ & $\mathrm{U}_{\mathrm{L}}$ & div. $W_{\text {TM }}$ & Qu \\
\hline & \multicolumn{4}{|c|}{ (in $1022 \mathrm{cal} / \mathrm{a}$ ) } & & & & \\
\hline & 6.3 & 0.35 & 1.55 & 8.2 & & & & \\
\hline Fall a & 5.67 & 0.32 & 1.86 & 7.85 & -10 & -10 & +20 & -4 \\
\hline Fall b & 5.04 & 0.28 & 2.32 & 7.64 & -20 & -20 & +50 & -7 \\
\hline Fall $\mathrm{c}$ & 5.04 & 0.70 & 2.32 & 8.06 & -20 & +100 & +50 & -2 \\
\hline Fall $\mathrm{d}$ & 5.04 & 0.70 & 2.46 & 8.2 & -20 & +100 & +59 & 0 \\
\hline Fall e & 5.04 & 0.42 & 2.74 & 8.2 & -20 & +20 & +77 & 0 \\
\hline Fall $\mathrm{f}$ & 4.41 & 0.70 & 3.09 & 8.2 & -30 & +100 & +99 & 0 \\
\hline Fall $\mathrm{g}$ & 4.41 & 0.25 & 3.79 & 8.45 & -30 & -30 & +144 & +3 \\
\hline
\end{tabular}

Bei den Fällen a, b und g setzen wir das Bowen-Verhältnis $U_{L} / U_{V}=$ const.; dies ist allerdings für die eiszeitlichen Kaltzeiten wohl kaum zutreffend, da bei einer Zunahme der advektiven Transporte div $W_{\text {TL }}$ notwendig die direkte Heizung der Atmosphäre von der Meeresoberfläche her $\left(U_{L}\right)$ auch wachsen muß. Bei Fall c nimmt zwar $U_{V}$ um $20 \%$ ab, aber $U_{L}$ auf das Doppelte zu; die resultierende Anderung von $Q_{M}$ liegt nur bei $-2 \%$. Gehen wir jetzt von der Annahme $\mathrm{Q}_{\mathrm{M}}=$ const. aus (Fälle d bis f), dann erkennen wir, daß eine sehr kräftige Erhöhung von $U_{L}$ und div $W_{T M}$ bei konstanter Strahlungsbilanz auch allein durch eine relativ geringe Verringerung der ozeanischen Verdunstung $U_{V}$ (um 20-30\%) geliefert werden kann (hierzu Kap. 4d). Da das Bowen-Verhältnis

$$
\frac{\mathrm{U}_{\mathrm{L}}}{\mathrm{U}_{\mathrm{V}}}=\frac{\mathrm{c}_{\mathrm{p}}}{\mathrm{L}} \cdot \frac{\partial \mathrm{T} / \partial \mathrm{z}}{\partial \mathrm{q} / \partial \mathrm{z}}
$$

( $c_{p}=$ spezifische Wärme der Luft bei konstantem Druck, $\mathrm{L}=$ Kondensationswärme) nur von den vertikalen Gradienten der Temperatur $\mathrm{T}$ und der spezifischen Feuchte $\mathrm{q}$ in der untersten Atmosphärenschicht abhängt, sind relativ kleine, advektiv bedingte Änderungen dieser Gradienten von ausschlaggebender Bedeutung für den Wärmehaushalt.

Andererseits bedeutet unsere Annahme $S_{0}=$ const. nicht notwendig auch eine Konstanz von Q.M. Die Gleichung der Strahlungsbilanz an der Meeresoberfläche lautet

$$
\mathrm{Q}_{\mathrm{M}}=(\mathrm{S}+\mathrm{H})\left(1-\mathrm{a}_{\mathrm{s}}\right)-(\mathrm{E}-\mathrm{G})
$$

Hierin bedeuten $\mathrm{S}+\mathrm{H}$ die einkommende Globalstrahlung als Summe der direkten Sonnenstrahlung $\mathrm{S}$ und der Himmelsstrahlung $\mathrm{H}$, während die langwelligen Komponenten E die Ausstrahlung der Meeresoberfläche $\left(\mathrm{E}=\sigma \mathrm{T}^{4}{ }_{\mathrm{s}}\right)$ und $\mathrm{G}$ die atmosphärische Gegenstrahlung (abhängig in erster Linie vom Wasserdampfgehalt der unteren Schichten) darstellen. Da E und $G$ unter kaltzeitlichen Klimabedingungen beide in der betrachteten Zone abnehmen müssen, läßt sich die Anderung der „effektiven“ Ausstrahlung E-G nur mit empirischen Formeln abschätzen. Bei einer Temperaturabnahme von $27^{\circ}$ auf $21^{\circ}$ und einer konstanten rel. Feuchte von $78 \%$ geht $\mathrm{E}-\mathrm{G}$ nach einem von GeIger gegebenen Diagramm von 44 auf $41 \mathrm{kLy} / \mathrm{a}$ zurück, d. h. QM nimmt entsprechend von 117 auf $120 \mathrm{kLy} / \mathrm{a}$ (um etwa $3 \%$ ) zu, wenn S + H konstant bleibt.

Die im Fall e angenommene Verdoppelung von div $\mathrm{W}_{\mathrm{TM}}$ reicht für die in einer Kaltzeit zu erwartenden advektiven Wärmeverluste hin, die - nach der in Kap. $2 \mathrm{f}$ erörterten extrem hohen Abkühlung in der Biskaya - im Kanarenstrom (Kap. 3 d) ihr Maximum erreichen. Nehmen wir für das Beispiel des tropisch-subtropischen Atlantiks wiederum an, daß der Wärmeaustausch zwischen ozeanischer Troposphäre und Stratosphäre durch die Thermokline vernachlässigbar klein ist und daß die mittlere zonale Geschwin- 
digkeit des Nord-Äquatorialstroms 5 Seemeilen $/ 24 \mathrm{~h}=10.7 \mathrm{~cm} / \mathrm{s}$ beträgt, so ist die mittlere Verweilzeit der Wassermassen in $10-20^{\circ}$ Breite zwischen Westafrika und dem Karibischen Meer etwa 2 Jahre. In dieser Zeit erhält eine $100 \mathrm{~m}$ mächtige Wassersäule von $1 \mathrm{~cm}^{2}$ Querschnitt $\left(=10^{4} \mathrm{~g}\right)$ insgesamt - wenn der Anteil von div $W_{\mathrm{TM}}$ an $\mathrm{Q}_{\mathrm{M}}$ von 0.19 sich verdoppelt $-2 \cdot 0.38 \cdot 120=91 \mathrm{kcal}$; das reicht aus, um diese Wassersäule um $9.1^{\circ}$ zu erwärmen. Damit kann also eine mittlere Temperaturdifferenz von $9^{\circ} \mathrm{zwischen}$ dem südwärts setzenden Kanarenstrom (vgl. Kap. $3 \mathrm{~d}$ ) und dem nordwärts setzenden Golfstrom stationär aufrecht erhalten werden, was etwa den kaltzeitlichen Verhältnissen entsprechen dürfte. Der heutige zonale Temperaturunterschied der beiden Stromsysteme beträgt nur $3-6^{\circ} \mathrm{C}$, was mit dem Wert für div $\mathrm{W}_{\mathrm{TM}}=0.19 \mathrm{Q}_{\mathrm{M}}$ gut übereinstimmt.

Aus diesen Rechnungen ergibt sich eine Wärmebilanz der tropischen Ozeanoberfläche, die schon bei geringen Anderungen der advektiv beeinflußten Gradienten $\partial \mathrm{T} / \partial \mathrm{z}$ und $\partial \mathrm{q} / \partial \mathrm{z}$ große Änderungen in dem gegenseitigen Verhältnis ihrer einzelnen Terme zuläßt, auch unter der Voraussetzung konstanter Zustrahlung. Aus dem Vorzeichen der Änderung der Wassertemperatur - die stets nur als kleine Differenz viel größerer Werte resultiert läßt sich keine Aussage über die wirksamen Vorgänge machen; eine Zunahme von div $W_{\mathrm{TM}}, \mathrm{d}$. h. des advektiven Wärmeentzuges durch Luft- und Meeresströmungen, um 75-100\% (Fälle e und $f$ ) kann schon durch eine Abnahme der Verdunstung um 20-30\% kompensiert werden (vgl. Kap. 4 d).

d) Eine von den bisherigen Betrachtungen grundsätzlich verschiedene Diskussion der kaltzeitlichen W a s e rbil a n z geht zweckmäßig aus (Flohn 1953) von der Dalton'schen Gleichung der Verdunstung V, in der diese lediglich als advektiver Prozeß (d. h. unabhängig von $Q_{M}$ ) behandelt wird:

$$
\mathrm{V}=\mathrm{ku}\left(\mathrm{e}_{\mathrm{s}}^{*}-\mathrm{e}\right)
$$

Hierin ist $\mathrm{k}$ eine Konstante, $\mathrm{u}$ die Windgeschwindigkeit, $\mathrm{e}^{*}$, der allein von der Oberflächentemperatur $\mathrm{T}_{\mathrm{s}}$ des Wasser abhängige Sättigungs-Dampfdruck an der Wasseroberfläche und e der aktuelle Dampfdruck der Luft. In unmittelbarer Nähe des Äquators etwa für die Zone $\varphi=0-5^{\circ}-$ ist die Windgeschwindigkeit u wegen des Verschwindens des horizontalen Coriolisparameters $\mathrm{f}=2 \Omega \sin \varphi(\Omega=$ Winkelgeschwindigkeit der Erdrotation, $\varphi=$ Breite) am Äquator selbst stets schwach: alle entstehenden Druckunterschiede werden binnen wenigen Stunden ausgeglichen. Da diese Verhältnisse auch in allen geologischen Vorzeiten anzunehmen sind, darf man $\mathrm{u}=$ const. als zeitunabhängig annehmen. Nehmen wir nunmehr - übrigens ebenso wie Manabe 1967 - Konstanz der relativen Feuchte $R F=e / e_{\mathrm{s}}$ an (heute $\mathrm{RF}$ über dem offenen Ozean $=78 \%$ ), dann ergibt sich folgender Vergleich (e und $\mathrm{e}^{*}$ in $\mathrm{mm} \mathrm{Hg}$ )

Tabelle 2

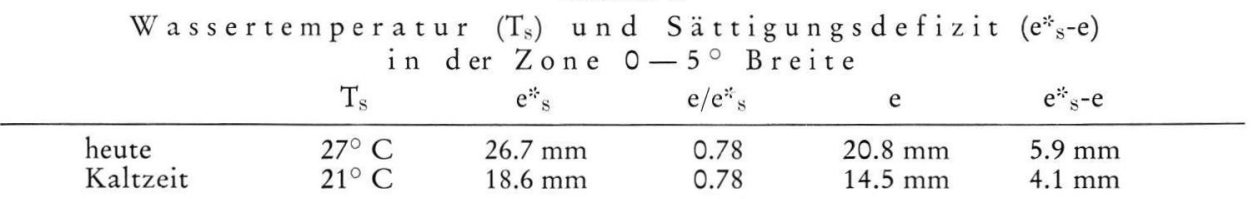

Trotz konstanter relativer Feuchte ist das Sättigungsdefizit $\mathrm{e}_{\mathrm{s}}-\mathrm{e}$ in einer Kaltzeit und damit auch die Verdunstung um $1.8 \mathrm{~mm}$ oder $30 \%$ geringer als heute (oder in einer anderen Warmzeit). Das stimmt also mit der Annahme der Fälle f und g (Kap. $4 \mathrm{c}$ ) überein. Andererseits erscheint eine erhebliche Änderung des Bowen-Verhältnisses $U_{L} / U_{V}$ nicht verträglich mit unserer Annahme RF = const. Aus diesem Grund wurde in Tabelle 1 noch der Fall g aufgenommen, der das Bowen-Verhältnis konstant läßt; da ein nicht vernachlässigbarer Teil der advektiven Transporte auch auf die Atmosphäre entfällt, ist diese Annahme wohl kaum streng gültig, aber bei der relativen Kleinheit von $U_{L}$ ohne Bedeutung, und Fall g mag eine Obergrenze für div $\mathrm{W}_{\mathrm{TM}}$ enthalten. 
$\mathrm{Da}$ heute in allen kalten Wintern das Azorenhoch und damit die atlantische Passatzirkulation geschwächt ist, können wir für die Kaltzeiten mit Sicherheit annehmen, daß die mittlere Windgeschwindigkeit $\mathrm{u}$ in der gesamten Tropenzone - sagen wir bis maximal $\varphi=30^{\circ} \mathrm{N}$ - nicht höher gewesen sein kann als heute. Dann ergibt sich für die tropisch-subtropische Meeresverdunstung, die heute im Gürtel $30^{\circ} \mathrm{N}-30^{\circ} \mathrm{S}$ mehr als $2 / 3$ der Gesamt-Verdunstung $\bar{V}$ der Erde liefert, eine Abnahme um mindestens 20-30\% in den Kaltzeiten, die (wegen $\bar{V}=\bar{N}$ ) von einer entsprechenden Verminderung der globalen Niederschlagsmenge $\bar{N}$ begleitet gewesen sein muß. Für eine hochglaziale (und spätglaziale) Trockenperiode gibt es so zahlreiche Belege - so besonders (Frenzel 1967) —, daß unsere Abschätzung jedenfalls dem Vorzeichen nach richtig sein muß. Nur in ganz wenigen Gebieten, wo die Verlagerung der Zyklonen-Zugbahnen die Abnahme des Wasserdampfgehaltes der Atmosphäre überkompensierte - etwa im Gebirgsstau in dem Teil des Mittelmeergebietes, wo die heutigen Winterregen zu einer ganzjährigen Regenperiode ausgeweitet wurden - waren die kaltzeitlichen Niederschläge höher als die heutigen. Diese kaltzeitliche Aridität belegt also auch die Realität der zunächst vielleicht überraschenden Annahmen unserer Modellfälle f und g. Eine weitere Ursache der globalen Abnahme von $\bar{V}$ und $\bar{N}$ in den Kaltzeiten liegt in dem Trockenfallen weiter Schelfflächen, besonders im Sundaarchipel, aber auch in anderen Randmeeren, wie Beringmeer und Nordsee, während Hudsonbay und Ostsee als Eisflächen gleichfalls nur noch sehr geringe Verdunstung lieferten. Das wird wohl kaum ganz kompensiert durch das Wachstum der Endseen in den Trockengebieten, wie Kaspi, Tschad und Lake Bonneville (Utah). Insgesamt ging der Meeresanteil an der Erdoberfläche von $71 \%$ auf etwa $68 \%$ zurück, was zu einem zusätzlichen Absinken von $\bar{V}$ um $3-4 \%$ führen müßte.

e) Bereits bei früheren Gelegenheiten (FLoHn 1967) wurde darauf hingewiesen, daß die bemerkenswerte rezente Asymmetrie der at mosphärischen Zirkulat io $n$ in Bezug auf den Äquator als Folge der ganz verschiedenen Wärmehaushalte der dünnen arktischen Meereisdecke einerseits, des antarktischen Festlandeises andererseits zu verstehen ist. Dieser thermische Gegensatz Arktis-Antarktis verschwindet im Nordwinter (Dezember-Januar) nahezu vollständig; dann fällt der meteorologische Äquator mit dem geographischen Aquator auch auf den Ozeanen zusammen. Er erreicht im Gegensatz hierzu eine nördlichste Grenzlage im Juli, wo das troposphärische Temperaturgefälle Äquator-Pol auf der Nordhemisphäre $17^{\circ}$, auf der Südhalbkugel $44^{\circ}$ beträgt; im Jahresmittel ist die antarktische Troposphäre $11-12^{\circ}$ kälter als die arktische.

Setzen wir die WiLson'sche Instabilitäts-Theorie des antarktischen Eises (WILSON 1964, 1966) als richtig voraus, dann ergibt sich die Frage nach ihren Konsequenzen für Wärmehaushalt und Zirkulation. Nach den oben mitgeteilten Abschätzungen ergibt sich eine mittlere Abkühlung der Südhalbkugel um $12^{\circ}$, die sich natürlich advektiv auch über den Äquator hinaus ausbreiten müßte. Auf jeden Fall vergrößert ein Schelfeisausbruch der Antarktis zunächst einmal das Kaltluftproduktionsgebiet und verschärft damit das meridionale Temperaturgefälle der Südhemisphäre, so daß der Gegensatz zur Nordhalbkugel noch vergrößert wird. Im Höhepunkt einer Kaltzeit hingegen vergrößert sich - jedenfalls im atlantischen Sektor $100^{\circ} \mathrm{W}-40^{\circ} \mathrm{E}$ - das meridionale Temperaturgefälle auf der Nordhalbkugel (südlich von $50^{\circ} \mathrm{N}$ ) ; hierdurch wird eine Abschwächung des hemisphärischen Gegensatzes und der äquatorialen Asymmetrie hervorgerufen, obwohl die primären Bedingungen des Wärmehaushaltes für den antarktischen Kontinent sich nicht ändern. Auf quantitative Abschätzungen muß hier verzichtet werden.

Die Frage nach der Rolle eines offenen, e is f reien a rkt is che $\mathrm{n} \mathrm{Ozeans}$ hat seit der Veröffentlichung der Eiszeit-Theorie von EwING und DonN (1956, 1958, 1966, 1968) eine erhebliche Rolle in der Diskussion gespielt. Für den zentralen Teil des arktischen Ozeans ist ein eisfreies Stadium in den letzten 25000 a, d. h. selbst in der postgla- 
zialen Wärmezeit ausgeschlossen (Ku \& BROECKER 1967). Obwohl wir für ältere Warmzeiten über keine direkten Bodenproben verfügen, lassen doch die palynologischen Befunde für Alaska/Kanada und Sibirien zur (vorletzten) Eem-Warmzeit (Frenzel 1967) eine allgemeine Erwärmung um $3-6^{\circ}$ gegenüber heute erkennen, verbunden mit einem starken Rückgang des Permafrostes (um über $600 \mathrm{~km}$ ) und einem Vorstoß der Waldgrenze (um bis $300 \mathrm{~km}$ ) nach Norden. Diese Befunde sprechen für eine erhebliche Verringerung der arktischen Treibeisfläche, sicher stärker als in der postglazialen Wärmezeit; doch ist ein Schluß auf ein völliges Verschwinden des Treibeises bisher noch nicht erlaubt. Die klimatischen Konsequenzen eines offenen arktischen Ozeans - Erhöhung der Schneemenge im Küstengebiet um einen Faktor 2-4 (vgl. heute Labrador oder Kamtschatka) sind jedoch m. E. mit der kaltzeitlichen Anordnung der Vereisungsgebiete (Kap. 2 f) nicht verträglich. Eine der weiteren Konsequenzen wäre, daß bei gleichzeitiger Aufrechterhaltung des antarktischen Festlandeises der Gegensatz der beiden Hemisphären und die äquatoriale Asymmetrie noch größer sein müßte als heute. Die Erwärmung der arktischen Troposphäre über einem eisfreien Ozean gegenüber den heutigen Verhältnissen hat RAKIPOW A (1966) auf $+3^{\circ}$ im Sommer, $+15^{\circ}$ im Winter geschätzt; auf mehr empirischer Grundlage ergibt sich für den Winter nur $+8-10^{\circ}$. Damit würde sich die ganzjährige Temperaturdifferenz Arktis-Antarktis auf nicht weniger als $17 \mathrm{bzw}$. $20^{\circ} \mathrm{C}$ erhöhen. Einige der damit gekoppelten Klimaänderungen wurden an anderer Stelle (FLETCHER 1965) behandelt.

\section{Aufbau eines synthetischen Modells}

In den folgenden Ausführungen soll in aller Kürze ein synthetisches Modell der pleistozänen Klimaschwankungen umrissen werden, das eine physikalische Interpretation der in Kapitel 2 dargestellten Befunde empirischer Natur ermöglichen soll. Ein Modell dieser Art muß notwendig auf viele, wichtig erscheinende Details verzichten; es darf andererseits zu quantitativen Abschätzungen nicht im Widerspruch stehen. Auf die Diskussion anderer Modelle und eine Stellungnahme zu einzelnen Hypothesen kann schon aus Raumgründen nicht eingegangen werden (vgl. SCHwarzbach 1968). Der Verfasser ist sich über manche kritischen Einwände durchaus klar; jedes Modell verträgt Änderungen, solange diese seine innere Konsistenz nicht gefährden und seine Übereinstimmung mit der Realität verbessern.

a) Polverlagerung und - hiervon weitgehend unabhängig - Drift der K o n t in e n t a l s cholle n lassen erkennen (Kap. 3a, b), daß sich seit dem frühen Tertiär die Rotationsachse der Erde langsam auf ihre jetzige Lage hin bewegte. Damit gerieten der weitgehend landumschlossene arktische Ozean, sowie das vom Indik aus südwärts driftende antarktische Festland in ihre jetzige Position. Diese ist charakterisiert durch eine weitgehende Isolierung (vom Weltmeer bzw. von den anderen Kontinenten) bei annähernd zirkumpolarer Lage: eine ähnliche Situation war offenbar während des ganzen Mesozoikums niemals gegeben. Hierbei ist die Position von Antarktika entscheidend; im Norden war die Verbindung zwischen arktischem Ozean und Atlantik niemals ganz unterbrochen - das muß betont werden, zumal im Tertiär zeitweise noch eine weitere Meeresverbindung über Westsibirien mit den tropisch-subtropischen Meeren bestand.

$\mathrm{Ab}$ Eozän (spätestens Oligozän) bildeten sich in Antarktika mit zunehmender An näherung an den Südpol zunächst ausgedehnte winterliche Schneefelder, deren Schmelzwässer am Rande des Kontinents die Meeresoberfläche abkühlten; durch Vermischung kam es im Verlauf einiger $10^{7}$ a zu einer Abkühlung der ozeanischen Tiefenzirkulation bis hin zum Bodenwasser der Aquatorregion (Emiliani 1955, 1957, 1961, 1966). Nehmen wir für die heutige Größe der Antarktis eine (nur geringe) Schmelzwassermenge von $1 \mathrm{~mm} / \mathrm{d}$ an, die mit $0^{\circ}$ dem Ozean (mit einer Temperatur von zunächst $10^{\circ}$ ) zugeführt wird. Das 
ergibt einen Wärmeentzug von $1.3 \cdot 10^{17} \mathrm{gcal} / \mathrm{d}$. Andererseits erhält der ozeanische Tiefenstrom (abgesehen von den sehr langsam wirkenden turbulenten Mischungsvorgängen) auf dem Ozeanboden von unten, vom Erdinnern her einen Wärmestrom von heute $0.13 \mathrm{Ly} / \mathrm{d}$; das sind bei einer Fläche der Ozeane südlich des Aquators von $206 \cdot 10^{6} \mathrm{~km}^{2}$ insgesamt $2.7 \cdot 1017 \mathrm{gcal} / \mathrm{d}$, also nur eben doppelt so viel wie der Wärmeentzug durch das Schmelzwasser. Diese Abkühlung - auch wenn sie sich nur auf die Schmelzperiode, also einen kleinen Teil des Jahres beschränkt - kann in geologischen Zeiträumen keinesfalls wirkungslos bleiben. Ohne Berücksichtigung anderer Wärmequellen reicht ein ständiger Wärmeentzug von $1.3 \cdot 1017 \mathrm{cal} / \mathrm{d}$ aus, um eine $1000 \mathrm{~m}$ mächtige Wasserschicht aller Ozeane bereits in 7500 a um $1^{\circ}$ abzukühlen; selbst wenn der Schmelzprozeß nur in jedem Jahr einen Monat anhält, benötigt die gleiche Abkühlung unter sonst konstanten Bedingungen knapp $10^{5}$ a. Durch seitliche und vertikale Vermischung kann nach Erreichen eines neuen Gleichgewichtszustandes im Laufe von $30-40 \mathrm{Ma}$ sicher eine weltweite Abkühlung der höheren Breiten um $8-10^{\circ}$ zustandekommen; dabei wird in der Tropenzone die geringmächtige warme Deckschicht oberhalb der Thermokline, die ständig eine Strahlungsbilanz von 250-300 Ly/d empfängt, zuletzt und am schwächsten betroffen. Die ozeanische Durchmischung ist wegen der geringeren Stabilität in den höheren Breiten stärker als in den Tropen: auf diese Weise nimmt das meridionale Temperaturgefälle $\partial \mathrm{T} / \partial \mathrm{y}$ sehr langsam zu.

Diese Zunahme erhöht - wie bereits früher ausgeführt (FLOHN 1964) - die thermische Rossby-Zahl und damit die Baroklinität der Atmosphäre. Dieser Effekt verursacht in der Theorie (FLOHN 1964) wie empirisch (Abb. 6) eine sehr allmähliche Verschiebung der subtropischen Hochdruckzone zum Aquator hin; lag sie in der warmen Periode des Alttertiärs noch in $50-60^{\circ}$ Breite (FLOHN 1964), so dürfte sie gegen Ende des Tertiärs bereits in etwa $40-45^{\circ}$ gelegen sein. Damit im Zusammenhang verstärken sich die beiden Polarwirbel mit ihrer Westdrift und weiten sich äquatorwärts aus; das Riesengebiet der von der tropischen oder Hadley-Zirkulation beherrschten Zone - vgl. Schwarzbach (1961), Abb. 105 - schrumpft allmählich auf etwa zwei Drittel des ursprünglichen Umfangs ein.

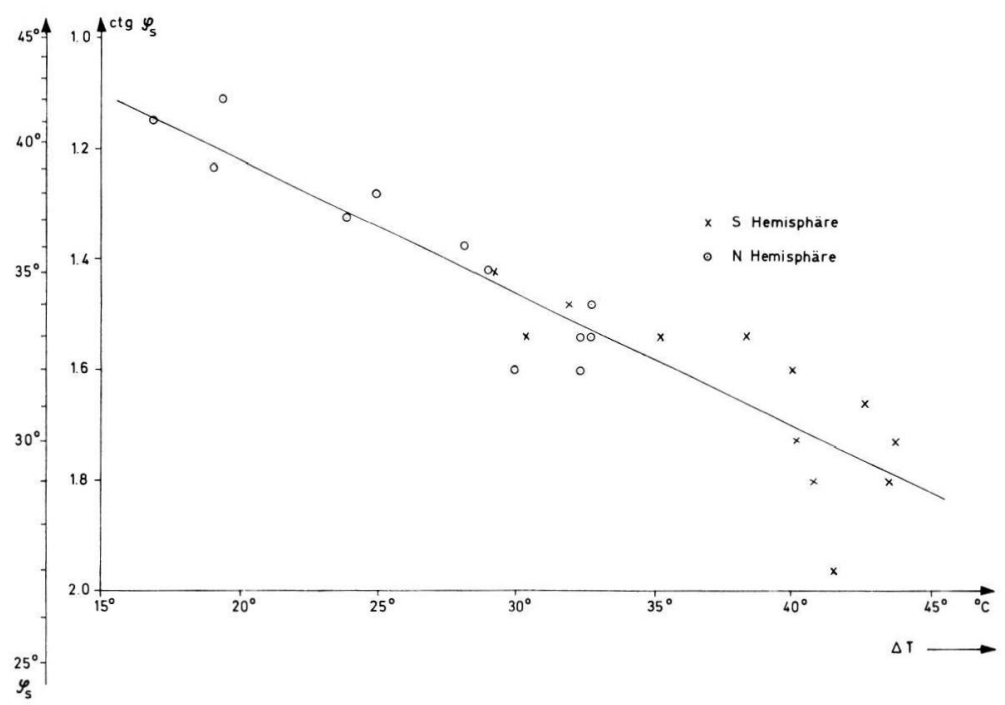

Abb. 6. Meridionales Temperaturgefälle $\Delta \mathrm{T}$ und Breitenlage $\left(\varphi_{\mathrm{s}}\right)$ der Subtropenzone (nach KoRFF) in einzelnen Monaten. 
Die Bildung von Gebirgsgletschern auf Antarktika steht sicher erst am Ende einer langen Entwicklung, bis die polaren Meere von $8-10^{\circ}$ bis in die Nähe des Nullpunktes abgekühlt waren; für unsere Überlegungen ist es sekundär, ob sie im Oligozän, im Miozän oder erst im Altpliozän erfolgt ist. Ihre Bedeutung besteht darin, daß nunmehr eine Abkühlung des subantarktischen Ozeans das ganze Jahr über aufrecht erhalten wird.

b) Im Laufe des Pliozäns ist die Abkühlung der höheren Breiten der Ozeane - hier die Bildung des antarktischen Kaltwassers zwischen dem antarktischen Kontinent und der antarktischen Konvergenz (Kap. $3 \mathrm{c}$ ) in $50-60^{\circ}$ Breite - soweit fortgeschritten, daß der a ntarktische Kontinent eine annähernd geschlossene Firneisdecke von zunächst geringer Dicke und stationärer Massenbilanz erhält. Da diese den Wärmenachschub von unten herabsetzt, wird ihre Oberfläche immer kälter, die sommerliche Schmelzperiode dauert immer kürzer, bis auch im Sommer die Oberflächenalbedo $a_{\mathrm{s}}$ von $80 \ldots 90 \%$ erhalten bleibt. Damit wird die Wärmequelle der sehr hohen sommerlichen Einstrahlung (heute $\mathrm{S}_{\mathrm{o}}$ bis $1180 \mathrm{Ly} / \mathrm{d}, \mathrm{S}+\mathrm{H}$ an der Erdoberfläche 800-900 Ly/d) weitgehend ausgeschaltet, und die winterliche Ausstrahlung führt den Übergang von einem warmen zu einem kalten Gletscher (Kap. 3 c) herbei, der die Massenverluste auf ein Minimum herabdrückt und zu einem (in geologischer Zeitskala) raschen Anwachsen führt. Nehmen wir (Hoinkes 1968, 1961) eine Massenbilanz von $+2 \mathrm{~cm} / \mathrm{a}$ (Wasserwert) an, dann benötigt das antarktische Eis $10^{5}$ a, um auf eine mittlere Mächtigkeit von 2200 m anzuwachsen. Dieser Prozeß muß aber gleichzeitig eine entsprechende eustatische Senkung des Meeresspiegels hervorrufen, von der uns im Pliozän nichts bekannt ist: wir dürfen ihn deshalb wohl nicht vor der Wende Pliozän/Pleistozän ansetzen.

Überschreitet das antarktische Inlandeis die Grenzen des antarktischen Kontinentes (heute $13.1 \cdot 10^{6} \mathrm{~km}^{2}$ ), bildet sich außer den kalbenden Eisbergen ein Schelfeis, dessen Wasserverdrängung bei Abschätzungen eustatischer Spiegelschwankungen nicht vernachlässigt werden darf. Sobald die Mächtigkeit den durch die Druckschmelzung gegebenen Schwellenwert überschreitet, kommt es im Sinne der Theorie Wilsons $(1964,1966)$ zu raschen Schelfeisausbrüchen. Nehmen wir mit WiLson eine Ausdehnung des Schelfeises bis $55^{\circ}$ Breite an, dann ergibt das eine Fläche von $33 \cdot 10^{6} \mathrm{~km}^{2}$; bei einer dem heutigen RoßEis entsprechenden Dicke von $300 \mathrm{~m}$ beträgt das Volumen des Schelfeis-Ausbruches $10 \cdot 10^{6} \mathrm{~km}^{3}$, im Vergleich zu dem heutigen Volumen des Antarktikeises von $28 \cdot 10^{6} \mathrm{~km}^{3}$. Mit diesem Ausbruch nimmt die mittlere Mächtigkeit des kontinentalen Eisschildes auf knapp $1400 \mathrm{~m}$ ab, bei einem eustatischen Meeresspiegelanstieg von $+25 \mathrm{~m}$. Spätestens in diesem Stadium gefriert das Inlandeis wieder als Ganzes, das Schelfeis bricht ohne Nachschub allmählich in Form riesiger Tafeln ab, und der kontinentale Eisschild beginnt wieder zu wachsen, umgekehrt proportional zu der Flächenausdehnung des randlichen Schelfeises. Unter der Annahme eines Massenzuwachses von maximal $+2-3 \mathrm{~cm} / \mathrm{a}$ ergibt sich ein quasiperiodischer Vorgang - Wachstum, Ausbruch und Abschmelzen - von der Dauer 30-50 000 a, d. h. in der gleichen Größe, wie sie durch die Befunde von Emiliani $(1955,1957,1961,1966)$ benötigt wird. (Eine Beteiligung der Erdbahnelemente an dieser Quasiperiodik ist möglich — vgl. WILson 1966 — aber offenbar nicht notwendig.)

Wilson's Annahme eines allseitigen, katastrophalen Ausbruches - der binnen weniger Jahre oder Jahrzehnte ablaufen müßte - erscheint wenig realistisch. Das Überschreiten der Schwelle vom „kalten“ zum „warmen“ Inlandeis mit der plötzlichen Abnahme der Viskosität hängt offenbar - auf die Theorie kann hier nicht näher eingegangen werden von zwei Variablen ab: Mächtigkeit und Mitteltemperatur des überlagernden Eises. Die Mächtigkeit des heutigen Antarktikeises schwankt zwischen 1 und $4 \mathrm{~km}$, das Jahresmittel seiner Oberflächentemperatur zwischen $-20^{\circ}$ und $-56^{\circ} \mathrm{C}$; hinzu kommt die vielgestaltige Orographie besonders im Küstenabschnitt. All dies spricht dafür, daß die von WILsoN 
angenommenen Schelfausbrüche in den einzelnen Sektoren zu sehr verschiedenen Zeiten erfolgen, zuerst vermutlich in den heutigen Schelfeisgebieten. Daraus ergibt sich nur eine allmähliche, vielleicht über 5-10000 a verteilte Wirkung auf das Klima, ebenso auch anstelle eines katastrophalen Anstieges des Meeresspiegels um 20-30 m eine ganze Reihe kleinerer Anstiege von wenigen Metern, vermutlich nur mit einer Rate von einigen $\mathrm{cm}$ (oder Dezimetern) pro Jahr. Auch die allmähliche Zunahme der Intensität der Kaltzeiten (Woldstedt 1966) läßt sich als Folge einer (überlagerten) Zunahme der Mächtigkeit des Antarktikeises bei gleichzeitiger Abkühlung interpretieren.

In diesem Zusammenhang darf kurz an die permoka rbone Eiszeit erinnert werden. Hier kam (schon im Devon) der riesige Gondwana-Kontinent, dessen Ausdehnung auf $65 \cdot 10^{6} \mathrm{~km}^{2}$ (also die fünffache Fläche von Antarktika) geschätzt werden kann, in Polnähe; über mehr als $100 \mathrm{Ma}$ hinweg wurden kälteliebende Faunen und Floren sowie lokale Vereisungen beobachtet. Im Permokarbon hat dieser Kontinent für längere Zeiten (20-30 Ma) annähernd zirkumpolar gelegen; in dieser Zeitspanne kam es zu einer kontinentalen Vereisung, die aber - schon wegen der durch die Größe des Kontinents bedingten Trockenheit - nie den ganzen Kontinent bis zur Küste umfassen konnte. Weil das Gondwana-Inlandeis stets kleiner als der Kontinent blieb, kam es nie zu quasiperiodischen Ausbrüchen und entsprechenden Meeresspiegelschwankungen; alle Vorgänge spielten sich im Innern des Kontinents ab, mit einer vergleichsweise sehr langsamen Zeitskala in der Größenordnung einiger $10^{7} \mathrm{a}$.

c) Der Wärmehaushalt der Erdoberfläche hat sich schon durch die allmähliche Abkühlung der Meere höherer Breiten erheblich gewandelt; die Schelfeis-Ausbrüche der Antarktis nach Wilson's Theorie liefern nun eine entscheidende Änderung (vgl. Kapitel 4 b) mit einer Zunahme von $a_{\mathrm{s}}$ (global) von rund 0.10 auf 0.15 und einem entsprechenden R ückgang der mittleren Oberflächentemperatur $\mathrm{T}_{\mathrm{s}}$ um $5-6^{\circ}$, beides jedoch konzentriert zunächst auf die höheren Südbreiten. Die physikalisch-klimatologischen Auswirkungen wären etwa die folgenden:

1. Da im atlantisch-afrikanischen Sektor $\left(45^{\circ} \mathrm{W}-75^{\circ} \mathrm{E}\right)$ die antarktische Konvergenz (als Folge der Konfiguration der Küsten) bis $49-50^{\circ} \mathrm{S}$ vorstößt, führt das Wachstum des antarktischen Schelfeises zuerst zu einer Abkühlung des Benguelastroms und des Südäquatorialstromes, der zum Teil in die nordhemisphärische Zirkulation, d. h. in den Golfstrom übertritt. Die konstante Strahlungszufuhr ( $\left.Q_{M}\right)$ in den Tropen schwächt zwar diese Abkühlung $a b$, aber im wesentlichen auf Kosten der Verdunstung $\left(U_{V}\right)$ des tropischen Meeres. Damit beginnt eine zwar langsame, aber nachhaltige Abkühlung von Luft und Wasser auch auf der Nordhalbkugel, die ein allmähliches Wachstum der Gletscher in Skandinavien, Labrador und Baffinland veranlaßt.

2. Wachstum der Inlandeise auf den Nordkontinenten und Vergrößerung der Treibeismenge auf dem Nordatlantik führen zu einer besonders großen Abkühlung des Kanarenstroms (lokal um etwa $12^{\circ}$, vgl. Kapitel $3 \mathrm{~d}, 4 \mathrm{c}$ ). Die annähernd konstante Einstrahlung der Tropenzone wird nunmehr zu einem wesentlich größeren Teil zur Erwärmung der oberen Schichten des Meeres verbraucht, so daß der Anteil der Terme $U_{V}+U_{L}$ an $\mathrm{Q}_{\mathrm{M}}$ von heute $80 \%$ auf wenig mehr als 50\% reduziert wird. Die Abkühlung der Meeresströmungen wird von einer Abkühlung der Luft begleitet, wenn auch stets der Wärmetransport $\mathrm{W}_{\mathrm{TL}}$ über den Ozeanen erheblich geringer bleibt als $\mathrm{W}_{\mathrm{TM}}$. Da von den tropischen Kontinenten (bei konstanter Einstrahlung) nur wärmere Luft auf das kalte Meere transportiert werden kann, nimmt nach der Dalton-Gleichung die Differenz $\mathrm{e}_{\mathrm{s}}$-e ab oder kehrt sogar ihr Vorzeichen um: dieser Vorgang setzt die Verdunstung (und $U_{V}$ ) erheblich herab, während $U_{L}$ vielfach sogar negativ wird. 
Die hier für den Atlantik ausgeführte Vorstellung gilt für den Pazifik nur in abgeschwächtem Maße, da wegen der Konfiguration des Kontinents und der Anordnung der Meeresströmungen die antarktische Konvergenz im pazifischen Sektor nur bis $58-60^{\circ} \mathrm{S}$ reicht. Der in Kapitel $2 \mathrm{f}$ erörterte große Unterschied der kontinentalen Vereisungen im atlantischen und pazifischen Sektor dürfte hierin primär mitbegründet sein. Insgesamt umfaßt der $\mathrm{A} \mathrm{ufbau}$ der E is s childe - ganz überwiegend im atlantischen Sektor der Nordkontinente $-32 \cdot 10^{6} \mathrm{~km}^{2}$ mit einer mittleren Mächtigkeit von $1600 \mathrm{~m}$ (Maximalwerte vielleicht $4000 \mathrm{~m}$ ). Der Spiegel des Weltmeeres sinkt gleichzeitig ab, in den größeren Eiszeiten auf $-100 \mathrm{~m}$, maximal auf $-145 \mathrm{~m}$. Während des Aufbaues sind die nordhemisphärischen Meerestemperaturen noch relativ hoch, ebenso auch Verdunstung und Niederschlag. Die Abkühlung der Kontinente führt zu Neubildung, Vergrößerung und Dickenwachstum der arktischen Meereisdecke: diese ist von ausschlaggebender Bedeutung für den regionalen Wärmehaushalt, aber auch für das meridionale Temperaturgefälle und damit für die Intensität der atmosphärischen Westdrift. Die Ausdehnung der Eisschilde bis $38^{\circ} \mathrm{N}$ (Mississippi) bzw. $48^{\circ} \mathrm{N}$ (Don-Wolga) erzwingt quasistationäre Höhentröge der Westdrift, die über dem nördlichen Südamerika und über Nordafrika bis in die inneren Tropen reichen: in diesen Gebieten kommt es zu besonders starkem Temperaturrückgang und (jedenfalls über der Sahara) zu höheren Niederschlägen.

Die Abkühlung der Luft und der Ozeanströmungen - jedenfalls im Kanarenstrom führen über der Aquatorialregion des Atlantik zu einem Temperaturrückgang um $5-6^{\circ}$ und (nach den in Kap. $4 \mathrm{c}$ erörterten Modellfällen) zu einem Rückgang der Verdunstung um rund 30\% : damit setzt die (annähernd stationäre) a ride $\mathrm{Ph}$ a se des $\mathrm{Hoch}$ g la zials ein, und das Wachstum der nordhemisphärischen Eisschilde endet. Die kontinentalen Eisschilde werden zunehmend von dem aus den ariden Gebieten stammenden Lößstaub verschmutzt, ihre Albedo nimmt ab, und damit wächst die Intensität (und Andauer) des sommerlichen Abschmelzens.

Dieser Vorgang leitet den $\mathrm{Abb}$ a u der beiden kontinentalen Eisschilde in Europa und Nordamerika ein, bleibt dagegen für Grönland und Antarktika unwirksam (HoINkes 1961, Bloch 1964, Davitaya 1965). Auch hierbei kann durchaus eine durch die Ekliptikschiefe hervorgerufene Zunahme der sommerlichen Einstrahlung (Milankovitch 1930, 1938, vaN WоERком 1953) unterstützend mitwirken.

Diese Hypothese beantwortet eine für den Meteorologen sehr schwierige Frage: wie kommt es zu der Umstellung von einer e is a $\mathrm{ufb}$ a e nden $\mathrm{Zirkulation} \mathrm{der} \mathrm{At-}$ mosphäre zu einer e is abba u enden ? Der erste Typ entspricht weitgehend (FloHN 1952) der heutigen Zirkulation kalter Winter (z. B. Winter 1962/3 oder 1968/9). Das ist voll verständlich, denn die im Wärmehaushalt so wichtige Grenze zwischen Schneedecke und schneefreiem Land (oder Meer) bestimmt über den thermischen Wind immer wieder die Konfiguration der Höhenströmung und damit die Zugbahnen der Zyklonen und Niederschlagsgebiete. Von ihrer Lage hängt es auch oft, in Nähe der $0^{\circ}$-Grenze, ab, ob Niederschlag als Regen oder als Schnee fällt. Je mächtiger die Eisschilde, desto persistenter ist diese Anordnung des troposphärischen Temperatur- und Windfeldes; blockierende HochZentren und tiefe Höhentröge sind jetzt nicht nur quasistationär, sondern durch die permanente Abkühlung über Eis fixiert. Die Zerstörung eines so weitgehend eingefahrenen Gleichgewichtes, das sich durch Rückkopplung (feed-back) immer wieder herstellt, ist vom synoptischen Gesichtspunkt heraus kaum verständlich: jeder Eisabbau benötigt eine völlige Umstellung dieser Anordnung, für die zunächst alle Voraussetzungen im dreidimensionalen Temperaturfeld fehlen.

Wird aber der Eisabbau unabhängig von der Wetterlage über den Strahlungshaushalt durch Abnahme der Albedo in Gang gesetzt, dann wird diese Rückkopplung an entschei- 
dender Stelle unterbrochen, und es kommt allmählich auch zu einer Umstellung der Großwettersituation. Das nordamerikanische Inlandeis ist - wenn wir die Verhältnisse der Postglazialzeit heranziehen (LAмв 1961, 1966) - stabiler als das europäische, dessen Abbau rascher vor sich geht; auch heute liegt der nordhemisphärische Kältepol der Troposphäre, besonders im Sommer, im Bereich des kanadischen Archipels. Ist aber das europäische Inlandeis erst einmal bis Skandinavien zurückgeschmolzen und das atlantische Treibeis bis $60^{\circ} \mathrm{N}$ zurückgewichen, dann verlagern sich die Zyklonenzugbahnen vom Mittelmeer nach Mitteleuropa (etwa 55 $5^{\circ}$ Breite), die lokale Abkühlung des Kanarenstroms entfällt, die äquatorialen Oberflächentemperaturen steigen rasch an — wie für die Zeit um 11000 vor heute wahrscheinlich gemacht - und eine der heutigen ähnlichere atmosphärische Zirkulation (LAMB 1961, 1966) setzt sich durch, obwohl das amerikanische Inlandeis in seinem Kern noch erhalten ist. Diese Phasenverschiebung erklärt also die rasche Erwärmung vor 11000 Jahren bei noch tiefem Stand des Meeresspiegels; vielleicht hängen damit auch die auffällig raschen und höchst wirksamen Klimaänderungen dieser Zeit (Schwarzbach 1961, Abb. 114) mit ihrer Zeitskala von nur 500-1000 a zusammen.

Mit dieser globalen Erwärmung des Oberflächenwassers geht die ebenso globale aride Phase zu Ende; mit der Zunahme der Temperatur und des Wasserdampfgehaltes der Atmosphäre etwa auf die heutigen Werte beschleunigt sich auch der Wiederaufbau des Antarktikeises.

d) Die besonders intensive Vereisung des zirkumatlantischen Sektors (Kap. $2 \mathrm{f}$ ) kann einmal primär darin begründet sein, daß die antarktische Konvergenz - an der Wilson $(1964,1966)$ mit guten Gründen seinen Schelfeis-Ausbruch enden läßt - im atlantisch-indischen Bereich $\left(45^{\circ} \mathrm{W}-75^{\circ} \mathrm{E}\right)$ in $48-50^{\circ} \mathrm{S}$ Breite liegt, gegenüber $60^{\circ} \mathrm{S}$ im pazifischen Sektor (Kap. 4 e). Damit kommt die initiale Abkühlung im Atlantik stärker zur Geltung als im Pazifik. Sobald aber die nordhemisphärischen Eisschilde erst einmal anwachsen und der Meeresspiegel unter $-20 \mathrm{~m}$ absinkt, verengt sich die Beringstraße stark; bei $-42 \mathrm{~m}$ ist sie völlig geschlossen. Damit verschwindet an dieser Stelle der Wärme- und Massenaustausch mit dem Arktischen Ozean, der heute etwa $35 \%$ des gesamten Austausches beiträgt. Die Warmwassermassen des Kuroshio verbleiben im Nordpazifik und führen zu einer mindestens relativen Erwärmung seines Nordabschnitts von den Kurilen bis zur Südküste von Alaska. Die dabei gewonnene Wärmeenergie beträgt nach Mosby etwa $8 \cdot 10^{19} \mathrm{cal} / \mathrm{a}$; dies reicht aus, um eine $100 \mathrm{~m}$ mächtige Deckschicht des Pazifiks nördlich $50^{\circ}$ Breite $\left(6.75 \cdot 10^{6} \mathrm{~km}^{2}\right)$ pro Jahr um $0,12^{\circ} \mathrm{zu}$ erwärmen. Das ist - neben dem vergleichsweise geringen hydrographischen Einzugsgebiet des Pazifiks offenbar eine der Hauptursachen für die auffällig geringe Vereisung von Nordost-Sibirien und Alaska. Während im Atlantik sich aus den Schmelzwässern an der Oberfläche eine flache, salzarme und daher wenig dichte Kaltwasserschicht bildet, noch verstärkt durch den Treibeis-Zustrom aus dem Arktischen Becken, fällt dieser Prozeß im Pazifik aus. Bis auf die globale - von der Breite mehr oder weniger unabhängige - Abkühlung als Folge der Zunahme der Albedo $a_{\mathrm{s}}$ (Kap. 4 b) ändert sich gegenüber den heutigen Verhältnissen nur wenig. Dagegen müßte im Atlantik als Folge der Vereisung beider Kontinente das a rktische Meereis in mehr oder minder aufgelockerter Form weit vorrücken von der Bäreninsel bis etwa zu einer Linie Irland-New York, also bis etwa $50^{\circ} \mathrm{N}$. Leider existiert kein Belegmaterial für oder gegen diese Vermutung; für die meteorologische Diskussion ist diese Frage zweifellos von entscheidender Bedeutung, worauf LAMB (1966) an mehreren Stellen hingewiesen hat. Die fundamentale Bedeutung der verschiedenen Wärmebilanzgrößen des arktischen Treibeises, des offenen Meeres nördlich $70^{\circ}$ Breite und im Vergleich dazu des antarktischen Festlandeises geht aus der folgenden Zusammenstellung (FLOHN 1967) hervor; sie darf allerdings für den kritischen Bereich zwischen $50^{\circ}$ und $70^{\circ} \mathrm{N}$ während der Kaltzeiten nicht ohne erhebliche Korrekturen angewandt werden. 
Tabelle 3

Strahlungs- und Wärmehaushalt von Arktis und Antarktis

\begin{tabular}{llrrrrrrr} 
& Jahreszeit & $\mathrm{Q}_{\mathrm{K}}$ & $\mathrm{Q}_{1}$ & $\mathrm{Q}$ & $\mathrm{U}_{\mathrm{V}}$ & $\mathrm{U}_{\mathrm{L}}$ & $\mathrm{U}_{\mathrm{M}}\left(\mathrm{U}_{\mathrm{B}}\right)$ & \\
\hline Arktischer & Sommer & 217 & -66 & 151 & 14 & 12 & 125 & $\mathrm{Ly} / \mathrm{d}$ \\
Ozean & Winter & 0 & -80 & -80 & -1 & -3 & -76 & $\mathrm{Ly} / \mathrm{d}$ \\
(eisbedeckt) & Jahr & 81.5 & -81 & 0.5 & 6.5 & 10 & -16 & $\mathrm{Ly} / \mathrm{d}$ \\
\hline Norwegische & Sommer & 312 & -79 & 233 & 26 & -17 & 224 & $\mathrm{Ly} / \mathrm{d}$ \\
See & Winter & 7 & -129 & -122 & 103 & 81 & -306 & $\mathrm{Ly} / \mathrm{d}$ \\
(eisfrei) & Jahr & 139 & -107 & 32 & 75 & 45 & -88 & $\mathrm{Ly} / \mathrm{d}$ \\
\hline Antarktischer & Sommer & 176 & -152 & 24 & 1 & 1 & 22 & $\mathrm{Ly} / \mathrm{d}$ \\
Kontinent & Winter & 0 & -50 & -50 & -1 & -40 & -9 & $\mathrm{Ly} / \mathrm{d}$
\end{tabular}

Hierbei ist $\mathrm{Q}_{\mathrm{K}}=(\mathrm{S}+\mathrm{H})\left(1-\mathrm{a}_{\mathrm{s}}\right)$ die kurzwellige, $\mathrm{Q}_{\mathrm{l}}=\mathrm{E}-\mathrm{G}$ die langwellige und $\mathrm{Q}=\mathrm{Q}_{\mathrm{K}}+\mathrm{Q}_{\mathrm{l}}$ die gesamte Strahlungsbilanz (Nettostrahlung) der Erdoberfläche. Ferner ist U der Wärmeumsatz bzw. (vertikale) Wärmetransport an der Erdoberfläche, mit den

Indizes $\mathrm{V}=$ Verdunstung, $\mathrm{L}=$ fühlbare Wärme (Luft), $\mathrm{B}=$ Boden, $\mathrm{M}=$ Meer.

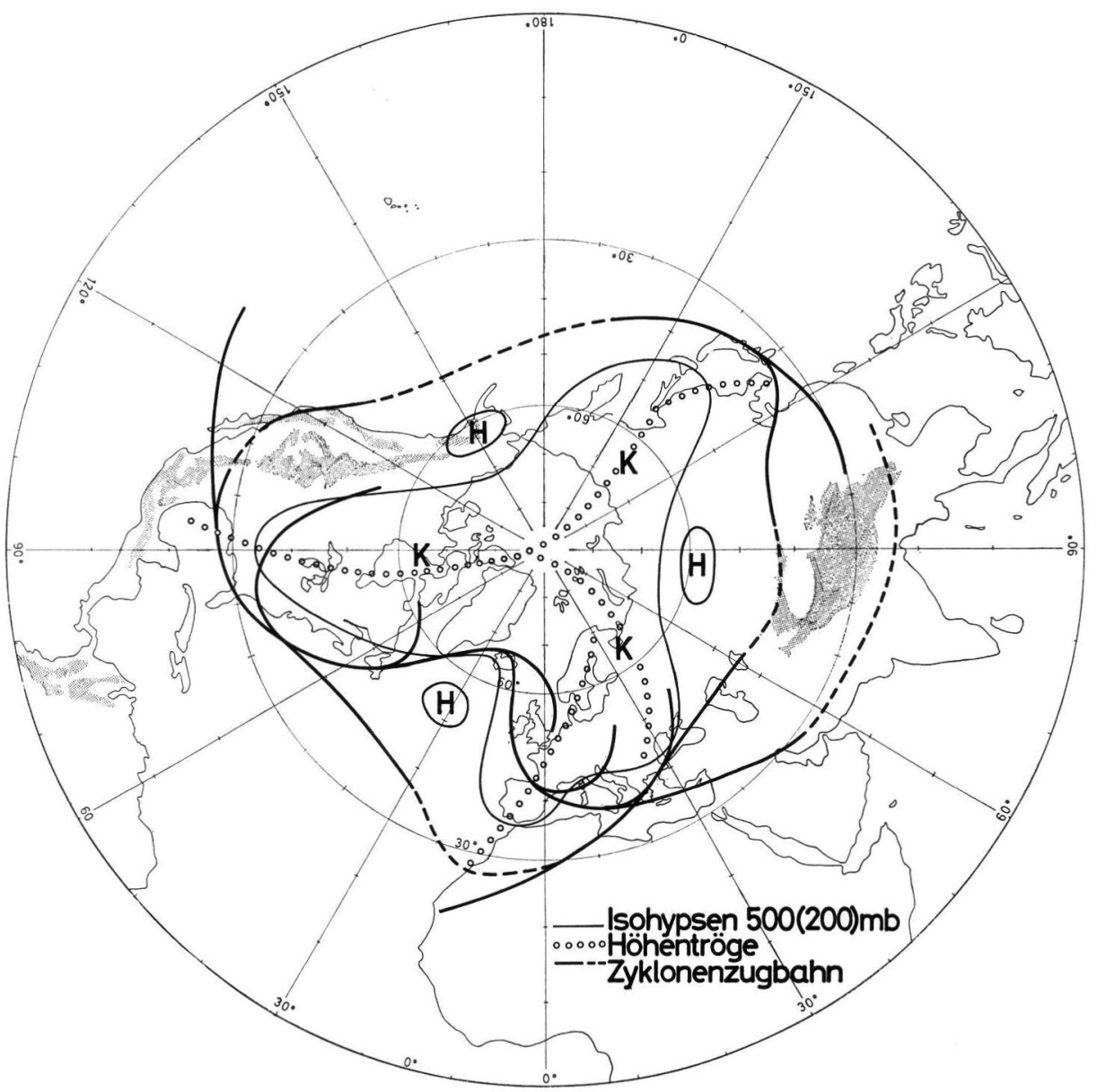

Abb. 7. Kaltzeitliche Anordnung der Höhenströmung (500-200 mb) und Zyklonenzugbahnen. 
Die besonders tiefe kaltzeitliche Absenkung der Temperaturen an der Westküste Europas von Irland bis nach Nordspanien um $12-15^{\circ}$ (Kap. 2 f) fordert eine spezielle Deutung, besonders beim Vergleich mit der amerikanischen Westküste in gleicher Breite. Da die nächsten Eisränder bei Irland, am Niederrhein (in der vorletzten Kaltzeit) und in den Westalpen liegen, scheidet eine Interpretation als Folge lokaler Gletscherwinde aus: deren thermische Wirkung geht kaum über $50-100 \mathrm{~km}$ hinaus. Wenn aber die Hauptzyklonenzugbahn - ebenso wie heute in allen Strengwintern - mit häufigen, intensiven Zyklogenesen in das Mittelmeer hinein verläuft, dann ergibt sich notwendig in Westeuropa ein Vorherrschen kalter östlicher Winde das ganze Jahr über. Diese Ostwinde müßten an der Biskaya mittels der Ekman-Drift eine Zone mit aufquellendem kalten Tiefenwasser in der inneren Biskaya hervorrufen, wobei die Wassertemperaturen - noch stärker als heute an der Küste von Oregon und Nordkalifornien - auf $8-10^{\circ} \mathrm{C}$ im Sommer absinken können, was nur noch zu einer subarktischen Tundra anstelle des Waldes im Meeresniveau ausreicht.

c) Die hypothetische Zirkulation der A t mosphäre während einer Kalt $z$ e it ist schon mehrfach Gegenstand meteorologischer Arbeiten zum Eiszeitproblem gewesen (Flohn 1952, Lamb 1961, 1966, Willett 1949); hierbei sind die Analogieen zu den heutigen Strengwintern an erster Stelle herangezogen worden. Nachdem etwa 30jährige Erfahrungen mit der heutigen dreidimensionalen Analyse vorliegen, sieht man in manchen Details klarer: die Dynamik der Rossby-Wellen (d.h. der Mäander der Westdrift) und die Rolle des thermischen Windes macht es relativ leicht, die vorherrschende Höhenströmung zu skizzieren (Abb. 7). Eine einwandfreie Darstellung der mittleren Bodendruckverhältnisse ist schon etwas schwieriger und mit mehr Fehlerquellen behaftet; wir verzichten deshalb hier darauf (vgl. jedoch LAMB 1966) und beschränken uns auf ein Schema der hauptsächlichen Zyklonenzugbahnen.

Zunächst darf man mit großer Wahrscheinlichkeit annehmen, daß die Hauptfrontalzonen, der subtropische Hochdruckgürtel und die mit ihm gekoppelte subtropische Strahlströmung im Atlantik ganzährig etwa $8-10^{\circ}$ weiter südlich lagen als heute, während im Pazifik diese Verlagerung mindestens geringer war. Das führt - nach der bekannten Formel von C. G. Rossby für die Länge $\mathrm{L}_{\mathrm{s}}$ stationärer Wellen in der Westdrift (vgl. Abb. 8)

$$
\mathrm{L}_{\mathrm{s}}^{2}=4 \pi \mathrm{U}^{2} / \beta \text { mit } \beta=2 \Omega \cos \varphi / \mathrm{r}
$$

( $\mathrm{U}=$ zonale Windgeschwindigkeit, $\Omega=$ Winkelgeschwindigkeit der Erde, $\varphi=$ Breite, $\mathrm{r}=$ Erdradius) - zu einer Verkürzung der quasistationären Wellen (LAмв 1966), sofern diese nicht durch die gleichzeitige Zunahme von $U$ überkompensiert wird. Aus der Analogie zu den heutigen Strengwintern ergibt sich ein quasistationärer tiefer Höhentrog im mittel- oder gar westeuropäischen Raum, in dem Kaltluft weit nach Nordafrika hineingeführt wird. An seiner Vorderseite kommt es zu häufigen und intensiven Niederschlägen

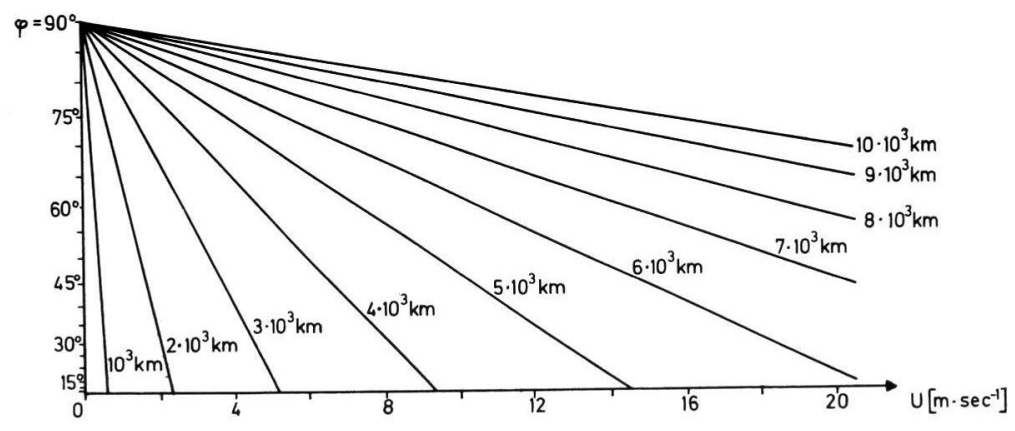

Abb. 8. Länge stationärer Rossby-Wellen. 
meist in Schneeform; auch in der Sahara treten - in Analogie zu den heute im Frühjahr dort häufigen Zyklogenesen - nicht unerhebliche Regenfälle ein. Dieser Höhentrog sichert die Ernährung des nordeuropäischen Inlandeises von SW, S und SE her bis hin nach Südrußland. Er ist notwendig gekoppelt mit der Tendenz zu blo ck i e r e n de n $\mathrm{Hochdruckgebieten} \mathrm{im} \mathrm{Seegebiet} \mathrm{um} \mathrm{Island,} \mathrm{aber} \mathrm{auch} \mathrm{auf} \mathrm{seiner} \mathrm{Vorderseite}$ im Raume Westsibirien $\left(60-80^{\circ} \mathrm{E}\right.$, etwa $\left.55^{\circ} \mathrm{N}\right)$ : damit erklärt sich auch die relative Trockenheit des Raumes Iran/Pakistan sowie die - im Vergleich zu den Alpen — geringe Vergletscherung der zentralasiatischen Hochgebirge, Mittel- und Ostsibiriens in der letzten Kaltzeit (v. Wissmann 1959). Der ostasiatische Höhentrog ist relativ schwächer als heute; hierfür spricht vor allem die begrenzte Vergletscherung der ostsibirischen Randgebirge. Das muß nicht in allen Kaltzeiten so gewesen sein: falls sich die sehr starke Vereisung von ganz Ostasien (auch Hochasien?) in einer älteren Eiszeit (Kap. $2 \mathrm{f}$ ) als gesichert herausstellen sollte, müssen wir mit erheblichen Verschiebungen in der relativen Bedeutung der einzelnen Höhentröge rechnen. Eine Ursache könnte darin liegen, daß in früheren Kaltzeiten die Beringstraße als Folge tektonischer Vorgänge nicht trocken fiel; damit wird wesentlich mehr Wärme dem Pazifik entzogen und dem Arktischen Ozean zugeführt. Doch erscheint eine eingehende Diskussion heute noch verfrüht.

Ein mittelatlantischer Höhenhochrücken - mit häufig blockierendem Zentrum bei Island - wird auch gefordert als Folge des sehr tiefen und beständigen Höhentroges über dem östlichen Nordamerika, dessen Achse in den Kaltzeiten wahrscheinlich etwas weiter im Westen $\left(85-90^{\circ} \mathrm{W}\right)$ lag als heute. Eine der wesentlichen Folgen ist eine große Häufigkeit nördlicher Winde auf seiner Vorderseite, d. h. vor den Küsten Westeuropas: das ist ein Argument mehr für eine relative Verstärkung des Kanarenstroms zu Lasten des Golfstromes, sowie für eine weit südwärts ausgreifende Zunge arktischen Treibeises. Eine weitere Folge ist die permanente Schwächung des Azorenhochs, des nordatlantischen Passats und damit der Wasserdampfzufuhr zum Pazifik, mit den (vgl. Kap. 3 d) - allerdings als primär! — skizzierten Folgen (WEYL 1968) für den Salzgehalt der beiden Ozeane.

\section{Zusammenfassung und Schluß}

Die Hauptergebnisse unserer Betrachtung können in folgenden Sätzen zusammengefaßt werden:

1) Die primäre Voraussetzung der pleistozänen (und der permokarbonen) Vereisung ist die Drift einer isolierten Kontinentalscholle - Antarktika im Tertiär, Gondwanaland im Devon - in eine zirkumpolare Position. Hieran sind im Tertiär sowohl konvektive Bewegungsvorgänge im Erdmantel (Spreizung der Ozeanböden) wie auch Verlagerungen der Rotationsachse relativ zum Erdmantel beteiligt; beide Prozesse sind nach Richtung und Geschwindigkeit durch paläomagnetische und (oder) astronomische Daten gesichert, mit einer horizontalen Komponente von wenigen $\mathrm{cm} / \mathrm{a}$.

2) Auf einem Kontinent in Polnähe bildet sich zunächst eine winterliche Schneedecke aus. Ihr Abschmelzen führt zur Abkühlung einer $1000 \mathrm{~m}$ mächtigen Bodenwasserschicht des Ozeans um $1^{\circ}$ in einem Zeitraum von $\sim 10^{5}-10^{6}$ a, die sich durch Mischungsvorgänge auf alle Ozeane ausdehnt, mit einem Minimum ihrer Wirkung in den warmen Deckschichten der Tropenzone. Dieser Effekt interpretiert die allmähliche Abkühlung der höheren Breiten während des Tertiärs, bei etwa konstanter Temperatur in den Tropen.

3) Spätestens im Pliozän bildet sich, nachdem die Meerestemperatur auf wenig über $0^{\circ}$ abgesunken war, auf Antarktika eine mehr oder minder stationäre Vergletscherung aus, vom Typ der „warmen“ Gebirgsgletscher. Gegen Ende des Pliozäns wandelt sich diese bei fortschreitender Abkühlung in einen „kalten“ Gletscher um, mit positiver Massenbilanz und gleichzeitigem eustatischen Absinken des Meeresspiegels; in den polaren Gebirgen der Nordkontinente bilden sich ebenfalls Gletscher. 
4) Sobald das antarktische Inlandeis die kritische Mächtigkeit erreicht, bei der auf der Unterseite Druckschmelzung eintritt, kommt es zu Ausbrüchen (surges) im Sinne der Theorie von A. T. Wilson, die am Rande des Kontinentes zu einer (allmählichen) Ausbildung großer Schelfeismassen bis etwa $55^{\circ} \mathrm{S}$-Breite führen, zugleich mit einem Anstieg des Weltmeeres von $20-30 \mathrm{~m}$. Die Zunahme der Oberflächenalbedo führt zu einer globalen Abkühlung um etwa $5^{\circ}$, zum Aufbau von Eisschilden in den dafür geeigneten Gebieten der Nordkontinente und damit zu eustatischem Absinken des Weltmeeres (maximal um $-145 \mathrm{~m})$.

5) Nach Eintritt der Druckentlastung stabilisiert sich das antarktische Inlandeis bei geringerer Mächtigkeit; das Schelfeis wird mangels Nachschub allmählich abgebaut.

6) Die von $\mathrm{N}$ und $\mathrm{S}$ her eingetretene Abkühlung der Meeresströmungen (und der Luft) führen in der Tropenzone zu einem advektiven Absinken der Meerestemperaturen um 5-6 $6^{\circ}$, zu einem Rückgang der Verdunstung um etwa $30 \%$ und damit (unter stationären Bedingungen) zu einer globalen ariden Phase im Hochglazial.

7) In dieser ariden Phase geht das Wachstum aller Eisschilde auf ein Minimum zurück; die Eisschilde der Nordkontinente werden durch Lößstaub so verschmutzt, daß das sommerliche Abschmelzen zunimmt und der Übergang zum Abbau (mit eustatischem Meeresspiegelanstieg im Spätglazial) einsetzt.

8) Die quasiperiodischen Vorgänge unter 4) bis 7) laufen mit einer Zeitskala von insgesamt 30-50000 a ab; die Mitwirkung von Schwankungen der Erdbahnelemente hierbei ist möglich, aber nicht notwendig.

9) Die viel stärkere Vereisung des atlantischen Sektors der Nordkontinente wird interpretiert (primär) als Folge des weiteren Ausgreifens der antarktischen Schelfeis-Ausbrüche im Südatlantik und (sekundär) als Folge der Bildung einer Landbrücke im Beringmeer, die den Massen- und Wärmeaustausch zwischen Pazifik und Arktis-Ozean verhindert.

10) Das Fehlen ähnlicher quasiperiodischer Vorgänge im Permokarbon kann interpretiert werden als eine Folge der Größe des Gondwana-Kontinentes, der eine Schelfeisbildung gar nicht zuläßt.

Diese 10 Sätze sind - um eine weitere Diskussion zu erleichtern - etwas überspitzt formuliert. Die damit skizzierte Modellvorstellung ist rein geophysikalis che r Natur (siehe jedoch Satz 8); die in ihr eingebauten geophysikalischen Theorieen stehen zwar nicht außerhalb jeder Diskussion, werden aber von vielen bedeutenden Autoren (besonders der englisch-sprechenden Welt) anerkannt.

Das Modell steht - soweit erkennbar - nirgendwo im Widerspruch zu Beobachtungstatsachen, die vielfach hier in ihren physikalischen Wechselbeziehungen erscheinen; dies erscheint als Vorzug gegenüber vielen „extraterrestrischen “ Eiszeit-Theorieen. Es benötigt weder eine Änderung der Solarkonstante noch (zwingend) eine Mitwirkung der Erdbahnelemente, weder eine Änderung der Gravitation noch eine solche der Zusammensetzung (oder Trübung) der Erdatmosphäre, abgesehen von dem temperaturabhängigen Anteil des Wasserdampfes. Eine derartige rein geophysikalische Deutung hält auch Hoinkes (1968) für möglich; vor 30 Jahren noch glaubte A. Penck (1938) fest an eine außerirdische Interpretation. Dabei erhält der antarktische Kontinent eine überragende Rolle als räumlich-zeitliches Steuerungszentrum (Sätze 1-5).

Der synthetische Charakter des Modells geht aus der Vielzahl der beteiligten Autoren hervor, von denen in vereinfachender Weise nur wenige genannt seien: zu Satz 1) FAIRbridge, sowie Heirtzler und Mitarbeiter, zu Satz 3) Hoinkes, zu Satz 4) Wilson sowie die grundlegend wichtigen Arbeiten von Manabe und Mitarbeitern, zu Satz 5) und 8) Wilson, zu Satz 7) Bloch, Davitaya und Hoinkes. Die Instabilitäts-Theorie von Wilson $(1964,1966)$ liefert (in etwas revidierter Form) den Auslöse-Mechanismus (Trigger), der innerhalb des terrestrischen Systems die Umschaltung von einem warmzeitlichen 
auf einen kaltzeitlichen Zustand bewirkt; auf ihre Prüfung muß also besonders Gewicht gelegt werden. Der Beitrag des Verfassers bezieht sich hauptsächlich auf die quantitativen Schätzungen zu Satz 2) und 6) sowie auf regionale Betrachtungen (u. a. Sätze 9-10).

Dieses empirisch-geophysikalische Modell hat - nach der Formulierung von ScHwarzвАСН (1968) - einen multilateralen, autozyklischen Charakter. Es soll die mathematische Behandlung paläoklimatischer Probleme fördern, wie sie MiLankovitch schon vor fast 50 Jahren - ohne Computer! - durchgeführt hat; eine sinnvolle mathematische Behandlung setzt eine solide Kenntnis der geophysikalischen Prozesse und Parameter voraus. Ein einfaches funktional-analytisches Paläoklima-Modell - bei dem die meridionale Verteilung der Temperatur am Boden vorgegeben wird - ist von einem meiner Mitarbeiter (Dipl.-Math. Fr. Sснміdт) entwickelt worden; sein weiterer Ausbau soll für einige der hier behandelten Probleme quantitative Lösungen liefern.

Der Verfasser ist vielen Gesprächspartnern für Diskussion und Kritik einzelner Fragen äußerst dankbar; stellvertretend für viele seien genannt: M. Bernard (New York), M. I. Budyko (Leningrad), P. Jordan (Hamburg), H. H. Lamb (Guildford, U. K.), M. Schwarzbach (Köln) und P. WOLDSTEDT (Bonn).

B e merkunge n bei der Korrektur: Wenn sich die kürzlich von Hars und Mitarb. (Bull. Geol. Soc. Amer., 80, 1481-1514, 1969) vorgelegte Neubestimmung des Alters verschiedener Bohrkerne aus der äquatorialen Tiefsee bestätigen sollte, dann ergibt sich eine neue Synthese der Datierung: 8 glaziale Epochen in den letzten 700000 Jahren, d. h. ein mittlerer Abstand von 80-90000 a. Diese (deutlich unperiodischen) Fluktuationen stehen m. E. nicht im Widerspruch mit dem hier vorgeschlagenen Modell, wohl aber mit der Milankovitch-Hypothese.

\section{Literatur}

Bernard, E. A.: Théorie astronomique des pluviaux et interpluviaux du quaternaire africain. Ac. roy. sc. d'outre-mer, mém. 80, 12, fasc. 1, 2-232, Brüssel 1962.

Bloch, M. R.: Die Beeinflussung der Albedo von Eisflächen durch Staub und ihre Wirkung auf Ozeanhöhe und Klima. - Geol. Rdsch., 54, 515-522, Stuttgart 1964.

Broecker, W. S.: Absolute dating and the astronomical theory of glaciation. - Science, 151, 299-304. Washington 1966.

- : Milankovitch hypothesis supported by precise dating of coral reefs and deep-sea sediments. Science, 159, 297-300, Washington 1968.

Budyко, M. I.: Atlas teplovogo balansa (Atlas der Wärmebilanz). - 41 S., Leningrad 1963.

Cox, A.: Polar wandering, continental drift, and the onset of quaternary glaciation. - Am. Met. Soc., Met. Monogr., 8, 30, 112-125, Boston, Mass. 1968.

Davitaya, F. F.: The possible influence of the atmosphere dusting on the decrease of glaciers and the climate warming (in russian). - Izv. Ak. Nauk USSR, Ser. Oeogr., 2, 3-22, Moskau 1965.

Doberitz, R., Flohn, H. \& Schütte, K.: Statistical investigations of the climatic anomalies of the equatorial Pacific. - Bonner Met. Abh., H. 7, 76 S., Bonn 1967.

Donn, W. L. \& EwING, M.: A theory of ice ages III. - Science, 152, 1706-1712, Washington 1966.

- : The theory of an ice-free arctic ocean. - Am. Met. Soc., Met. Monogr., 8, 30, 100-105, Boston 1968.

Emiliani, C.: Pleistocene temperatures. - J. Geol., 63, 538-578, Chicago, Ill. 1955.

Emiliani, C. \& Geiss, J.: On glaciations and their causes. - Geol. Rdsch., 46, 576-601, Stuttgart 1957.

Emiliani, C.: Cenozoic climatic changes as indicated by the stratigraphy and chronology of deepsea cores of globigerina - ooze facies. - Ann. New York Ac. Sc., 95, 521-536, New York 1961.

- : Isotopic palaeotemperatures. - Science, 154, 851-857, Washington 1966.

Ericsson, D. B., Ewing, M. \& Wollin, G.: The pleistocene epoch in deep-sea sediments. - Science, 146, 723-732, Washington 1964.

- : Pleistocene climates and chronology in deep-sea sediments. - Science, 162, 1227-1234, Washington 1968.

EwIng, M. \& Donn, W. L.: A theory of ice ages I. - Science. 123, 1061-1066, Washington 1956.

- : A theory of ice ages II. - Science, 127, 1159-1162, Washington 1958. 
FAirbridge, R. W.: Convergence of evidence on climatic change and ice ages. - Ann. New York Ac. Sc., 95, 542-579, New York 1961.

-: Ice-Age Meteorology. Ice Age Theory. In: Encyclopedia of Astrogeology, 454-474, New York 1967.

Fletcher, J. O.: The heat budget of the arctic basin and its relation to climate. - Rand Corp., R-444-PR, 179 S., Santa Monica, Cal. 1965.

FLINT, R. F.: Glacial and pleistocene geology. - 553 S., New York 1957.

Flohn, H.: Allgemeine atmosphärische Zirkulation und Paläoklimatologie. - Geol. Rdsch., 40, 153-178, Stutgart 1952.

- : Studien über die atmosphärische Zirkulation in der letzten Eiszeit. — Erdkunde, 7, 266-275, Bonn 1953.

- : Kontinental-Verschiebungen, Polwanderungen und Vorzeitklimate im Lichte paläomagnetischer Meßergebnisse. - Naturw. Rdsch., 12, 375-384, Stuttgart 1959.

- : Grundfragen der Paläoklimatologie im Lichte einer theoretischen Klimatologie. - Geol. Rdsch., 54, 504-515, Stuttgart 1964.

- : Bemerkungen zur Asymmetrie der atmosphärischen Zirkulation. - Ann. Met. (N.F.), 3, 76-80, Offenbach M. 1967.

Flohn, H. \& KorfF, H. Cl: Z Zusammenhang zwischen dem Temperaturgefälle Äquator-Pol und den planetarischen Luftdruckgürteln. - Ann. Meteor. N. F., 4, 163-164, Offenbach a. M. 1969.

Frechen, J. \& Lippolt, H. J.: Kalium-Argon Daten zum Alter des Laacher Vulkanismus, der Rheinterrassen und der Eiszeiten. - Eiszeitalter u. Gegenwart, 16, 5-30, Ohringen 1965.

Frenzel, B.: Die Klimaschwankungen des Eiszeitalters. - 291 S., Braunschweig 1967.

Geiger, R.: Das Klima der bodennahen Luftschicht. - 4. Aufl., S. 23 ff., Braunschweig 1961.

Gow, A. J., Ueda, H. T. \& Gartfield, D.: Antarctic ice sheet. - Science, 161, 1011-1013, Washington 1968.

HAys, J. D.: Quaternary sediments of the antarctic ocean. In SEARS, M. (ed.): Progress in Oceanography, 4, 117-131, Oxford 1967.

Hays, J. D. \& Opdyke, N. D.: Antarctic radiolaria, magnetic reversals, and climatic change. Science, 158, 1001-1011, Washington 1967.

Heusser, C. J.: Polar hemispheric correlation: Palynological evidence from Chile and the Pacific north-west of America. In Roy. Met. Soc.: World climate from 8000 to O B.C. Proc. Int. Symp. London 1966, 124-141, London 1967.

Hoinkes, H.: Die Antarktis und die geophysikalische Erforschung der Erde. - Naturwissenschaften, 48, 354-374, Berlin 1961.

- : Wir leben in einer Eiszeit. - Umschau, 68, 810-815, Frankfurt M. 1968.

Jordan, P.: Die Expansion der Erde. - 182 S., Braunschweig 1966.

KaIsER, Kh.: Das Klima Europas im quartären Eiszeitalter. - Fundamenta, Monogr. z. Urgesch., 2, 1-27, Köln 1967.

Kerner von Marilaun, F.: Paläoklimatologie. - Berlin 1930.

KLebelsberg, R. von: Handbuch der Gletscherkunde und Glazialgeologie. - 1 u. 2, Wien 1948/9.

Ku, T. L. \& Broecker, W. S.: Rates of sedimentation in the arctic ocean. In SEARS, M. (ed.): Progress in Oceanography, 4, 95-104, Oxford 1967.

LAmb, H. H.: Fundamentals of climate. In NAIRN, A. E. M. (ed.): Descriptive palaeoclimatology, 8-44, New York 1961.

- : The changing climate. Selected papers. 236 S., London 1966.

Lamb, H. H., Lewis, R. P. W. \& Woodroffe, A.: Atmospheric circulation and the main climatic variables between 8000 and O B.C.: Meteorological evidence. In Roy. Met. Soc.: World climate from 8000 to O B.C. Proc. Int. Symp. London 1966, 174-217, London 1967.

Laue, E. G. \& Drummond, A. J.: Solar constant: First direct measurement. - Science, 161, 888891, Washington 1968.

Manabe, S. \& STrickler, R. F.: Thermal equilibrium of the atmosphere with a convective adjustment. - J Atm. Sc., 21, 361-384, Boston 1964.

Manabe, S. \& Wetherald, R. T.: Thermal equilibrium of the atmosphere with a given distribution of relative humidity. - J Atm. Sc., 24, 241-259, Boston 1967.

Milankowitch, N.: Mathematische Klimalehre und astronomische Theorie der Klimaschwankungen. In KöPpEN, W. \& Geiger, R.: Hdb. Klimat., 1, A, Berlin 1930.

Mrtchell, J. M. jr.: Theoretical paleoclimatology. - In: Quaternary of the U. S., Review Vo. for the VII Congr. Int. Ass. f. Quatern. Res., 881-901, Princeton 1965.

Mossy, H.: Water, salt and heat balance of the north polar sea and of the Norwegian sea. - Geof. Publ., 24, 289-313, Oslo 1962.

ỚPIK, E. J.: Climatic change in cosmic perspective. - Icarus, 4, 289-307, New York 1965.

Penck, A.: Die Strahlungstheorie und die geologische Zeitrechnung. - Z. Ges. Erdk. Berlin, H. 9/10, 321-350, Berlin 1938. 
Pitman, W. C. III, Herron, E. M. \& Heirtzler, J. R.: Magnetic anomalies in the Pacific and sea floor spreading. - J. Geophys. Res., 73, 2069-2136, Richmond 1968.

Rakipova, L. R.: The influence of the arctic ice cover on the zonal distribution of atmospheric temperature. - Rand Corp. Proc. Symp. Arct. Heat Budget and Atm. Circul., 31. Jan.-4. Febr. 1966 Lake Arrowhead, Cal., Memorand. RM-5233-NSF, 411-441, Santa Monica, Cal. 1966.

RASCHKE, E.: The radiation balance of the earth-atmosphere system from radiation measurements of the Nimbus II meteorological satellite. - NASA, TN D-4589, 81 S., Washington, D. C. 1968.

Runcons, S. K.: Changes in the convection pattern in the earth's mantle and continental drift: Evidence for a cold origin of the earth. - Symp. Continental Drift, Roy. Soc. Phil. Transact., 258, No. 1088, London 1965.

Schwarzbach, M.: Das Klima der Vorzeit. - 2. Aufl., 275 S., Stuttgart 1961.

- : Neuere Eiszeithypothesen. - Eiszeitalter u. Gegenwart, 19, 250-261, Öhringen 1968.

Schwind, M.: Das japanische Inselreich. - 1, 333-337, Berlin 1967.

Sellers, W. D.: Physical climatology. - 272 S., Chicago 1965.

SHAw, D. M. \& DonN, W. L.: Milankovitch radiation variations: A quantitative evaluation. Science, 162, 1270-1272, Washington 1968.

Stoyко, A.: Mouvement séculaire du pôle et la variation des latitudes des stations du Sil. In Markowitz, Wm. \& Guinot, B. (ed.): Continental drift, secular motion of the pole, and rotation of the earth. - Int. Astron. Union, Symp., 32, 52-56, Dordrecht 1968.

Sтоуко, N.: Variation séculaire des longitudes. In Markowitz, Wm. \& Guinot, B. (ed.): Continental drift, secular motion of the pole, and rotation of the earth. - Int. Astron. Union, 32, 57-62, Dordrecht 1968.

VINE, F. J.: Spreading of the ocean floor: New evidence. - Science, 154, 1405-1416, Washington 1966 .

WEYL, P. K.: The role of the oceans in climatic change: A theory of the ice ages. - Am. Met. Soc., Met. Monogr., 8, 30, 37-62, Boston 1968.

Willett, H. C.: Long-period fluctuations of the general circulation of the atmosphere. - J Met., 6, 34-50, Boston 1949 .

WILSON, A. T.: Origin of ice ages: An ice shelf theory for pleistocene glaciation. - Nature, 201, 147-149, London 1964.

- : Variation in solar insolation to the south polar region as a trigger which induces instability in the antarctic ice sheet. - Nature, 210, 477-478, London 1966.

Wissmann, H. von: Die quartäre Vergletscherung in China: Die heutige und jungeiszeitliche Vergletscherung in den Randgebieten Chinas. - Z. Ges. Erdk. Berlin, 241-262, Berlin 1937.

- : Die heutige Vergletscherung und Schneegrenze in Hochasien, mit Hinweisen auf die Vergletscherung der letzten Eiszeit. - Ak. Wiss. u. Lit. Mainz, Abh. math.-naturw. Kl., Jg. 1959, 14, 1101-1431, Wiesbaden 1959.

Woerkom, A. J. J. van: The astronomical theory of climate changes. In Shaplex, H.: Climatic change, 147-157, Cambridge, Mass. 1953.

Woldstedt, P.: Das Eiszeitalter. Grundlinien einer Geologie des Quartärs. - 1, 3. Aufl., 2 u. 3, 2. Aufl., Stuttgart 1958, 1961, 1965.

- : Der Ablauf des Eiszeitalters. - Eiszeitalter u. Gegenwart, 17, 153-158, OOhringen 1966.

Wund, W.: Das Reflexionsvermögen der Erde zur Eiszeit. - Met. Z., 55, 81-87, Braunschweig 1938.

- : Der Energiehaushalt der Erde im Laufe des Jahres und in der Erdgeschichte. - Met. Z., 56, 325-329, Braunschweig 1939.

- : Die Mitwirkung der Erdbahnelemente bei der Entstehung der Eiszeiten. - Geol. Rdsch., 34, 713-747, Stuttgart 1944.

Manuskr. eingeg. 27. 5. 1969.

Anschrift des Verf.: Prof. Dr. H. Flohn, Meteorologisches Institut der Univ., Bonn, Auf dem Hügel 20. 